\title{
Hippocampal Lesion Prevents Spatial Relational Learning in Adult Macaque Monkeys
}

\author{
Pamela Banta Lavenex, ${ }^{1,2}$ David G. Amaral, ${ }^{1}$ and Pierre Lavenex ${ }^{1,2}$ \\ ${ }^{1}$ Department of Psychiatry and Behavioral Sciences, Center for Neuroscience, California National Primate Research Center, The Medical Investigation of \\ Neurodevelopmental Disorders Institute, University of California, Davis, Sacramento, California 95817, and 2Institute of Physiology, Department of \\ Medicine, University of Fribourg, 1700 Fribourg, Switzerland
}

The role of the hippocampus in spatial learning and memory has been extensively studied in rodents. Comparable studies in nonhuman primates, however, are few, and findings are often contradictory. This may be attributable to the failure to distinguish between allocentric and egocentric spatial representations in experimental designs. For this experiment, six adult monkeys received bilateral hippocampal ibotenic acid lesions, and six control subjects underwent sham surgery. Freely moving monkeys then foraged for food located in two arrays of three distinct locations among 18 locations distributed in an open-field arena. Multiple goals and four pseudorandomly chosen entrance points precluded the monkeys' ability to rely on an egocentric strategy to identify food locations. Monkeys were tested in two conditions. First, local visual cues marked the food locations. Second, no local cues marked the food locations, so that monkeys had to rely on an allocentric (spatial relational) representation of the environment to discriminate these locations. Both hippocampal-lesioned and control monkeys discriminated the food locations in the presence of local cues. However, in the absence of local cues, control subjects discriminated the food locations, whereas hippocampal-lesioned monkeys were unable to do so. Interestingly, histological analysis of the brain of one control monkey whose behavior was identical to that of the experimentally lesioned animals revealed a bilateral ischemic lesion restricted to the hippocampus. These findings demonstrate that the adult monkey hippocampal formation is critical for the establishment or use of allocentric spatial representations and that selective damage of the hippocampus prevents spatial relational learning in adult nonhuman primates.

Key words: allocentric; declarative memory; medial temporal lobe; hippocampus; CA1; selective lesion

\section{Introduction}

Since the description of the amnesic patient H.M. (Scoville and Milner, 1957), researchers have worked to define the precise role of the medial temporal lobe structures in learning and memory. Although there is still significant debate about the exact nature of the cognitive processes subserved by these structures (Mishkin et al., 1998; Squire and Zola, 1998; Tulving and Markowitsch, 1998; Brown and Aggleton, 2001), a prominent theory advocates that the hippocampal formation (comprising the dentate gyrus, hippocampus, subiculum, presubiculum, parasubiculum, and entorhinal cortex) (Lavenex and Amaral, 2000) is essential for the processing of relational information (Cohen and Eichenbaum, 1993; Eichenbaum, 2000; Buckmaster et al., 2004).

One aspect of hippocampal function that has been studied extensively in rodents is its role in spatial relational learning and memory, i.e., the formation and use of allocentric representa-

Received Dec. 19, 2005; revised March 1, 2006; accepted March 21, 2006.

This research was conducted at the California National Primate Research Center (National Institutes of Health Grant RR00169) and was supported by a Faculty Alumni Research Development Fund grant from the Department of Psychiatry and Behavioral Sciences at University of California, Davis, National Institute of Health Grants R01NS16980 and R01-MH57502, and Swiss National Science Foundation Grant PP00A-106701.

Correspondence should be addressed to Dr. Pierre Lavenex, Institute of Physiology, University of Fribourg,

Chemin du Musée 5, CH-1700 Fribourg, Switzerland. E-mail: pierre.lavenex@unifr.ch.

DOI:10.1523/JNEUROSCI.5412-05.2006

Copyright $\odot 2006$ Society for Neuroscience $\quad 0270-6474 / 06 / 264546-13 \$ 15.00 / 0$ tions of space (O'Keefe and Nadel, 1978; Nadel and Hardt, 2004). Numerous studies have linked the integrity of the hippocampal formation to a rodent's ability to learn and remember a particular location in the absence of local cues marking that location, i.e., in relation to distant environmental cues (Morris et al., 1982; Nadel, 1991; Schenk et al., 1995). Electrophysiological studies have further implicated the rodent hippocampus in spatial learning and memory (O'Keefe and Dostrovsky, 1971; O'Keefe and Nadel, 1978; Nadel, 1991; Eichenbaum, 1999). Similarly, aided by advances in functional magnetic resonance imaging (MRI), neuropsychologists have begun investigating the role of the human hippocampal formation in spatial learning and memory (Morris and Mayes, 2004). Following the theoretical framework developed from studies in other animals, recent studies have confirmed that there are multiple forms of spatial knowledge and suggest that the human hippocampal formation is particularly involved with allocentric (viewpoint-independent), but not egocentric (viewpoint-dependent), representations of space (Astur et al., 2002; Bohbot et al., 2004; Parslow et al., 2004; Shelton and Gabrieli, 2004).

Comparable studies on the role of the nonhuman primate hippocampal formation in spatial memory, however, are few, and findings are often contradictory. For example, whereas several studies have demonstrated that hippocampal-lesioned monkeys exhibit impaired performance in various spatial memory 
tasks (Angeli et al., 1993; Beason-Held et al., 1999; Malkova and Mishkin, 2003; Hampton and Murray, 2004; Alvarado and Bachevalier, 2005), other studies have failed to show any spatial memory impairment after selective hippocampal lesions (Ridley et al., 1997; Murray and Mishkin, 1998; Malkova and Mishkin, 2003). Nevertheless, electrophysiological studies suggest that the primate hippocampus is involved in spatial learning and memory (Rolls et al., 1989; Ono et al., 1991; Rolls and O'Mara, 1995; Georges-Francois et al., 1999; Hori et al., 2003). We suggest that monkey hippocampal lesion experiments have produced inconsistent results because many of the tasks that have been used can be solved by using allocentric (hippocampal-dependent) or egocentric (hippocampal-independent) representations of space (Nadel and Hardt, 2004). Appreciation of this distinction, however, is absolutely critical to decipher the exact role of the primate medial temporal lobe structures in spatial learning and memory (Lavenex and Banta Lavenex, 2006).

The aim of this study was to determine whether the adult monkey hippocampal formation is critical for the establishment or use of allocentric (spatial relational) representations of space.

\section{Materials and Methods}

All protocols were approved by the Institutional Animal Care and Use Committee of the University of California, Davis, and were in accordance with the National Institutes of Health guidelines for the use of animals in research.

\section{Experimental subjects}

Subjects were 12 adult (10- to 13-year-old) male macaque monkeys ( $\mathrm{Ma}$ caca mulatta). Monkeys were born and raised at the California National Primate Research Center (CNPRC). They were maternally reared in 2000 $\mathrm{m}^{2}$ outdoor enclosures and lived in large social groups until $\sim 1$ year before surgery (i.e., at 6-9 years of age). At that time, each adult male was moved indoors and maintained in a standard home cage $[61 \mathrm{~cm}$ width $(\mathrm{W}) \times 66 \mathrm{~cm}$ depth $(\mathrm{D}) \times 81 \mathrm{~cm}$ height $(\mathrm{H})$ ]. Subjects were originally conscripted for a study on the role of the amygdala in social behavior, in which the hippocampal-lesioned and sham-operated subjects used here served as controls (and thus received identical treatment). For 1 year before the lesion and sham surgeries, and for the 42 months after the surgeries, monkeys' behavioral responses were observed while they were exposed to a variety of animate and inanimate stimuli, including heterospecific animal-like stimuli, live conspecifics, and humans. After surgery, monkeys were also tested in a potentiated startle paradigm that concluded 4 months before the beginning of these experiments.

For these experiments, monkeys were not subjected to any food or water restriction except that they did not receive their regular morning rations until after testing was completed. Monkeys were tested at the same time each day ( $5 \mathrm{~d} /$ week), between 7:30 and 10:30 A.M.

\section{Stereotaxic surgery}

Presurgical magnetic resonance imaging. To define exact lesion coordinates, monkeys underwent MRI. Subjects were anesthetized with ketamine hydrochloride ( $15 \mathrm{mg} / \mathrm{kg}$, i.m.) and medetomidine $(25-50 \mu \mathrm{g} / \mathrm{kg}$, i.m.) and imaged using a $1.5 \mathrm{~T}$ Gyroscan magnet (GE Healthcare, Little Chalfont, Buckinghamshire, UK); 1.0-mm-thick sections were created using a T1-weighted inversion recovery pulse sequence (repetition time, $21 \mathrm{~ms}$; echo time, $7.9 \mathrm{~ms}$; number of excitations, 3; field of view, $8 \mathrm{~cm}$; matrix, $256 \times 256$ ). From these images, a stereotaxic atlas was created for each individual animal to determine the precise location of the hippocampus and calculate the coordinates for the injections of ibotenic acid. The intent of the injection was to make a complete lesion of the dentate gyrus, hippocampus, subiculum, presubiculum, and parasubiculum without damaging surrounding brain regions.

Surgical procedures. All surgical procedures were performed aseptically at the CNPRC. Monkeys were first anesthetized with ketamine hydrochloride (15 mg/kg, i.m.) and medetomidine (25-50 $\mu \mathrm{g} / \mathrm{kg}$, i.m.). A stable level of anesthesia was maintained throughout surgery with a com- bination of isoflurane (1.0\%, inhalation; varied as needed to maintain an adequate level of anesthesia) and intravenous infusion of fentanyl (7-10 $\mu \mathrm{g} \cdot \mathrm{kg}^{-1} \cdot \mathrm{hr}^{-1}$, i.v.). Monkeys were ventilated, and their vital signs were monitored throughout surgery. A midline scalp incision was made, the skin was displaced laterally, and the skull was exposed. Bilateral craniotomies were made over the hippocampus, and the dura was retracted to expose the surface of the brain. Electrophysiological recordings were made to confirm the dorsoventral coordinates of the injection sites. A tungsten microelectrode was lowered into the hippocampus at a midrostrocaudal, midmediolateral position, and recordings from salient features of the hippocampus were documented and used to adjust the injection coordinates. Simultaneous bilateral ibotenic acid $(10 \mathrm{mg} / \mathrm{ml}$ in 0.1 м PBS; Biosearch Technologies, Novato, CA) injections were placed into the hippocampus using $10 \mu$ l Hamilton syringes (26 gauge beveled needles) at a rate of $0.2 \mu \mathrm{l} / \mathrm{min}$. We made a total of 12-18 injections and used a total of 10-16 $\mu \mathrm{l}$ of ibotenic acid, bilaterally, for each monkey. After injections, the dura was sutured, the craniotomy was filled with Gelfoam (Pfizer, New York, NY), and the fascia and skin were sutured in two separate layers. After the surgical procedure, monkeys were monitored by a veterinarian until they were able to be returned to their home cages. Six sham-operated control monkeys were anesthetized as described above and received midline scalp incisions, and their skull was exposed. Control subjects were maintained under anesthesia for the average duration of the lesion surgeries, and the fascia and skin were sutured in two separate layers. Analgesics $(0.15 \mathrm{mg} / \mathrm{kg}$ oxymorphone given three times daily for $2 \mathrm{~d}$ ) were administered postsurgically, and a prophylactic regimen of antibiotics (50 $\mathrm{mg} / \mathrm{kg}$ Clarofan, three times daily) was administered for the first $5 \mathrm{~d}$ after surgery.

\section{Open-field apparatus}

The open-field arena was constructed at the CNPRC and consisted of a hexagonal board (made of white acrylic plastic, $210 \mathrm{~cm}$ in diameter) placed in a large Plexiglas enclosure $[220 \times 220 \mathrm{~cm}$ (Fig. 1)]. Monkeys entered and exited the arena from side-mounted wire-mesh holding chutes via one of four entrances at the corners of the arena (see below). Eighteen plastic cups $(7.5 \mathrm{~cm}$ in diameter) were arranged in a regular pattern on the hexagonal board. The board was mounted on wheels, which allowed it to be rotated about its central axis (see below). The plastic cups were inverted so that monkeys had to lift or turn them over to obtain a food reward. The arena was surrounded by three clear Plexiglas panels [front panel, roof panel, and top half of the back panel (Fig. 1, dashed lines)], allowing a clear view of distant environmental cues distributed in the experimental room (Fig. $1 A$ ). Objects permanently located in the experimental room for the duration of testing included three doors or openings located on one side of the room: one door connecting the experimental room to another experimental room, one opening leading to a storage space, and one door leading to the outside of the building. A fourth opening on the other side of the room connected the experimental room to the temporary housing room containing a rack of 12 holding cages in which monkeys waited immediately before and after testing: a flood lamp $(100 \mathrm{~W})$ facing the testing apparatus was attached to the top right corner of the Plexiglas enclosure (on the outside); a yellow "sun" ( $30 \mathrm{~cm}$ in diameter) painted on a blue tarp ("sky," $220 \mathrm{~cm} \mathrm{~W} \times 120$ $\mathrm{cm} \mathrm{H})$; a metallic pole supporting the roof of the room (5 $\mathrm{cm}$ in diameter); a brown desk $(120 \mathrm{~cm} \mathrm{~W} \times 70 \mathrm{~cm} \mathrm{D} \times 70 \mathrm{~cm} \mathrm{H})$; an American flag $(95 \mathrm{~cm} \mathrm{~W} \times 50 \mathrm{~cm} \mathrm{H})$; a "tree" painted on a gray tarp $(120 \mathrm{~cm} \mathrm{~W} \times 180$ $\mathrm{cm} \mathrm{H})$; a digital camera mounted on a tripod $(120 \mathrm{~cm} \mathrm{H})$; a metallic transfer cage $(30 \mathrm{~cm} \mathrm{~W} \times 50 \mathrm{~cm} \mathrm{D} \times 42 \mathrm{~cm} \mathrm{H})$ used to transport the monkeys between the temporary housing cages and the holding chutes at the beginning and the end of each session (always placed at the same location while the monkey was in the testing arena); and the observer (always sitting at the same location). Fluorescent lights attached to the walls of the room directly above the tree and the American flag provided additional lighting. Vertically sliding doors at each corner of the arena could be remotely operated (from the front of the apparatus), allowing the monkeys to go in and out of the arena from the wire-mesh holding chutes located along both sides of the arena. Opaque side panels (Fig. 1, solid lines) provided visual barriers between the open-field arena and the wire-mesh chutes. Monkeys had full access to distant environmental cues 
A

Experimental Room

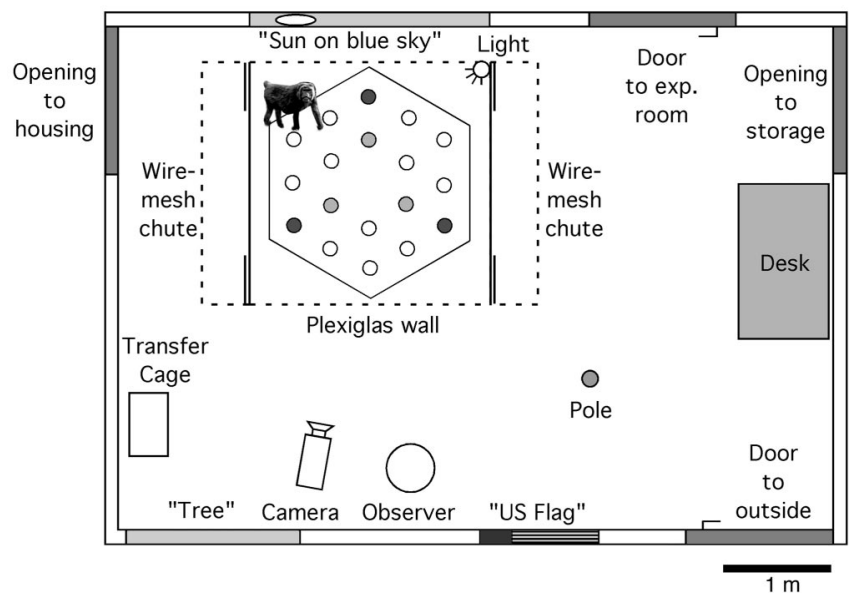

B

Experimental Conditions

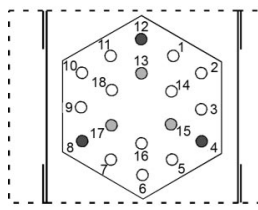

Local Cue

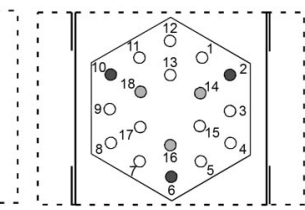

Probe Trial

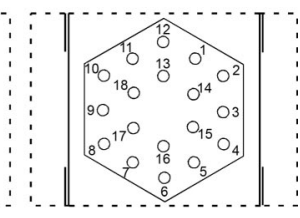

Spatial Relational
Figure 1. Schematic representation of the testing environment and experimental conditions. $A$, Aerial view of the experimental room. Eighteen plastic cups were regularly distributed on a hexagonal board $(210 \mathrm{~cm}$ in diameter) placed in a square testing arena $(220 \mathrm{~cm} \mathrm{~W} \times 220$ $\mathrm{cm} \mathrm{D} \times 220 \mathrm{~cm} \mathrm{H}$ ). Remotely operated sliding doors at each corner of the arena (double solid lines) allowed the animals to go in and out of the arena from wire-mesh chutes located along both sides. The front panel, the roof, and the top half of the back panel (dashed lines) were made of Plexiglas, allowing clear view of distant environmental cues; two opaque side panels (solid lines) provided visual barriers between the open-field arena and the wire-mesh holding chutes. The ability of monkeys to rely on an egocentric representation of space was precluded by alternating pseudorandomly between four different entrances into the open-field arena and using multiple goal locations. $\boldsymbol{B}$, Schematic representation of the arena in the different testing conditions. (1) Local cue condition: blue cups marked potentially-baited locations 4, 8, 12 on the outer array, and red cups marked potentially-baited locations $13,15,17$ on the inner array. All other locations were covered with neutral (beige) cups. (2) Probe trial: colored cups were shifted $60^{\circ}$ from the correct spatial locations. Blue cups were at locations $2,6,10$, and red cups were at locations $14,16,18$. Neutral cups were at locations $4,8,12$ and $13,15,17$, as well as at locations $1,3,5,7,9,11$. No food was present. (3) Spatial relational condition: neutral cups covered the potentially-baited locations $4,8,12$ and $13,15,17$, as well as all other locations.

from within the arena and from the holding chutes (Fig. 1). All testing was videotaped with a remotely controlled camera located in front of the testing apparatus.

\section{Procedure}

Pretraining and acclimation. Monkeys began pretraining and acclimation 42 months after surgery (i.e., when they were $10-13$ years of age). Monkeys were first trained to displace a plastic cup to retrieve a marshmallow hidden underneath during a pretraining phase that took place in the temporary holding cages $(61 \mathrm{~cm} \mathrm{~W} \times 66 \mathrm{~cm} \mathrm{D} \times 81 \mathrm{~cm} \mathrm{H})$. They received 20 trials per day until they successfully retrieved the fully covered marshmallow in $<30 \mathrm{~s}$ on all trials; this phase lasted up to $5 \mathrm{~d}$. After pretraining, monkeys received one 5-min acclimation session per day for $5 \mathrm{~d}$, during which they were free to explore the open-field arena. During this phase, there was no food or cups present in the arena. The acclimation phase was aimed at habituating the monkeys to the testing environment before the beginning of actual testing.

Testing. Freely moving monkeys foraged for a preferred food (marshmallows) located in two different arrays of three distinct locations (an inner array and an outer array) (Fig. 1). Monkeys were given three trials per day (with a $1 \mathrm{~min}$ intertrial interval), $5 \mathrm{~d}$ /week. Monkeys behaved freely, without any negative reinforcement to shape their behavior. Trials were terminated either $30 \mathrm{~s}$ after a monkey had found all three baited cups or at $5 \mathrm{~min}$ (whichever came first). Monkeys were coaxed in and out of the open-field arena from holding chutes that ran the length of the testing apparatus on either side. From the holding chutes, the monkeys were unable to see the arena while the experimenter replenished the food under the cups. On each trial, monkeys entered and exited the arena from one of the four pseudorandomly chosen entrances (see below). The orientation of the entire apparatus remained fixed in relation to distant environmental cues within the testing room. The board on which the cups were distributed was rotated clockwise $60^{\circ}$ before each trial to make any noncontrolled local cues, such as olfactory traces, irrelevant, but the marshmallows (and local cues if present) were always placed in exactly the same locations in relation to distant environmental cues (Fig. 1) (Lavenex and Schenk, 1998; Lavenex and Banta Lavenex, 2006). Each day, three locations on either the inner array or the outer array were baited. The location of the food changed pseudorandomly between the two arrays between sessions ( $24 \mathrm{~h}$ interval) but remained the same between trials ( 1 min interval) within a daily session. On the outer array, only cups at locations 4,8 , and 12 could be baited. On the inner array, only cups at locations 13,15 , and 17 could be baited. The other locations on the inner array $(14,16,18)$ and on the outer array $(1,2,3,5,6,7,9,10$, 11) were never baited.

The experiment included two different testing conditions: a local cue condition and a spatial relational condition. The experiment was conducted in four successive phases to assess potential effects of continual testing on the monkeys' abilities: monkeys were first tested for 3 weeks in the local cue condition, followed by 3 weeks in the spatial relational condition, followed by 2 weeks in the local cue condition, followed by 2 weeks in the spatial relational condition.

Local cue condition. In this condition, monkeys were tested to assess their ability to find food at fixed locations marked by local cues, i.e., colored cups (Fig. $1 B$ ). Blue cups marked the potentially-baited locations on the outer array (locations 4, 8, 12), and red cups marked the potentially-baited locations on the inner array (locations 13, 15, 17). Neutral (beige) cups covered all other locations $(1,2,3,5,6,7,9,10,11$, $14,16,18)$. The particular array that was baited remained the same within a daily session but changed pseudorandomly between days. However, regardless of which array was baited, both sets of local cues (i.e., blue and red cups) were present on the board during all local cue sessions. Thus, in the local cue condition, monkeys could use either local cues or spatial relational information to discriminate the locations of the food. Monkeys received a total of 5 weeks of experience ( $5 \mathrm{~d} /$ week) with three trials each day (i.e., $25 \mathrm{~d}$ or 75 trials) in the local cue condition (weeks 1, 2, 3 and 7,8 ).

Dissociation probe trial. After each testing phase in the local cue condition, monkeys were tested on a single probe trial during which the locations of the colored cups were shifted $60^{\circ}$ from their usual spatial locations and no food was present (Fig. $1 B$ ). This probe trial took place as the first trial of the day (on days 16 and 41) and was followed by three standard trials in the spatial relational condition (see below). The aim of this probe trial was to assess the monkeys' reliance on the local cues versus spatial relational information to discriminate potentially-baited locations from never-baited locations (Lavenex and Banta Lavenex, 2006). During standard local cue trials, potentially-baited locations were covered by a colored cup, so that both local and spatial relational information were coherent. During the dissociation probe trial, however, colored cups covered the never-baited locations 2, 6, 10, 14, 16, 18, and neutral (beige) cups covered the normally potentially-baited locations 4 , $8,12,13,15,17$, as well as the never-baited locations $1,3,5,7,9,11$. As monkeys entered the arena, they encountered the same pattern of cups distributed throughout the arena, but the absolute locations of the colored cups within the arena were shifted, thus rendering the local and spatial relational information incoherent. The monkeys were faced with two competing strategies to determine where the food might be hidden (although no food was actually present for this trial), either (1) under the 
colored cups at spatially "incorrect" locations, or (2) under neutral cups at "correct" spatial locations.

Spatial relational condition. In this condition, no local cues (i.e., no colored cups) marked the potentially-baited locations, i.e., all locations were covered by neutral cups (Fig. $1 B$ ). Therefore, monkeys could not discriminate between potentially-baited and never-baited locations based on local features. Instead, monkeys had to rely on a spatial relational representation of the environment to discriminate these locations, i.e., they needed to form an allocentric representation of space that coded the goal locations in relation to distant environmental cues (Lavenex and Banta Lavenex, 2006). Monkeys received a total of 5 weeks of experience ( $5 \mathrm{~d}$ /week) with three trials per day (i.e., $25 \mathrm{~d}$ or 75 trials) in the spatial relational condition (weeks 4, 5, 6 and 9, 10). The combination of multiple goal locations (six) and four pseudorandomly chosen entrance points precluded the ability of monkeys to rely on an egocentric strategy to identify food locations.

\section{Data analysis}

Data analysis included weeks $2,3,7$, and 8 (i.e., $20 \mathrm{~d}$ or 60 trials) in the local cue condition and weeks 5, 6, 9, and 10 (i.e., $20 \mathrm{~d}$ or 60 trials) in the spatial relational condition. We did not include weeks 1 and 4 , the first weeks of testing in the local cue and spatial relational conditions, respectively, to decrease the amount of variability that was attributable to the animals learning the procedural aspects of the task.

To identify the strategies that control and hippocampal-lesioned monkeys used to discriminate the baited locations, each of the 18 locations was classified into one of five categories with respect to whether it could be baited and its position in the open-field arena (Lavenex and Banta Lavenex, 2006). Thus, "Pot IN" denoted the three potentially-baited locations at the corners of the inner hexagon (Fig. 1, locations 13, 15, 17); "Pot OUT" denoted the three potentially-baited locations at the corners of the outer hexagon (locations 4, 8, 12); "Equ IN" denoted the three never-baited locations at the corners of the inner hexagon (locations 14, 16,18 ; termed "equivalent" because of their position at one of the three corners of the hexagon, topologically equivalent to the position of the potentially-baited locations); "Equ OUT" denoted the three never-baited locations at the corners of the outer hexagon (locations 2, 6, 10); and "Other" denoted the never-baited locations on the sides of the outer hexagon (locations $1,3,5,7,9,11$ ). In this way, we could analyze not only the monkeys' performance (i.e., whether they were efficient at discriminating baited locations) but also identify which cognitive strategies monkeys were able, or unable, to use to guide their behavior. In the local cue condition, monkeys could rely on a visual guidance strategy (egocentric coding), because local cues (colored cups) identified the food locations. In contrast, in the spatial relational condition, the reliance on an egocentric representation of space might enable the discrimination of locations on the inner array from locations on the outer array, but it does not allow the discrimination of locations on the same array. Instead, monkeys must remember the food locations in relation to distant environmental cues to be able to discriminate Pot IN from Equ IN locations or Pot OUT from Equ OUT locations, which are located at topologically equivalent locations at the corners of the inner and outer hexagon, respectively (Lavenex and Banta Lavenex, 2006). Therefore, we can infer that monkeys that choose more Pot IN than Equ IN locations or more Pot OUT than Equ OUT locations rely on an allocentric representation of space to guide their behavior. In contrast, monkeys that choose as many Equ IN locations as they do Pot IN locations and as many Equ OUT locations as they do Pot OUT locations do not rely on an allocentric representation of space to guide their behavior.

The evaluation of choice types for the first choice (i.e., the first cup opened during an individual trial), the first four choices (i.e., the first four cups opened during an individual trial), the first six choices (i.e., the first six cups opened during an individual trial), or for all choices (i.e., all of the cups opened during an individual trial) made by each monkey allowed us to further assess the behavioral strategies exhibited by individual subjects (Lavenex and Banta Lavenex, 2006). For each analysis, we normalized the number of choices of a particular type based on the probability to make that choice (Lavenex and Banta Lavenex, 2006). For standard trials, the number of choices of Pot IN (locations 13, 15, 17), Pot
OUT (locations 4, 8, 12), Equ IN (locations 14, 16, 18), and Equ OUT (locations 2, 6, 10) was divided by three, and the number of Other choices (locations 1, 3, 5, 7, 9,11) was divided by six. For probe trials, the number of choices of "Color IN" (locations 14, 16, 18), "Color OUT" (locations 2, $6,10)$, "Space IN" (locations 13, 15, 17), and "Space OUT" (locations 4, 8,12 ) was divided by three, and the number of Other choices (locations $1,3,5,7,9,11$ ) was divided by six. We report the first and first four choices for all standard trials, and the first six and all choices made during the dissociation probe trials.

Statistical analyses were conducted using ANOVAs, with lesion condition as a factor and choice type as repeated measures. Post hoc analyses were performed with Fisher's PLSD tests. Within-group analyses were conducted with choice type as repeated measures (Lavenex and Schenk, 1995, 1996, 1997, 1998; Lavenex et al., 1998; Lavenex and Banta Lavenex, 2006). Because monkeys might exhibit unique strategies to find the food locations (Lavenex and Banta Lavenex, 2006), we also analyzed the behavior of each individual monkey separately to detect evidence of learning. In this case, statistical analyses were performed within subjects, with choice type as a factor and daily sessions as repeated measures. Statview 5.0.1 statistical software (SAS Institute, Cary, NC) was used to perform all statistical analyses.

We want to note that one of the sham-operated monkeys (MMU26736) exhibited behavioral strategies identical to those of experimentally lesioned monkeys. Histological examination of its brain revealed a bilateral lesion restricted to the hippocampus that is described extensively at the end of Results. This monkey was therefore excluded from the control group. All behavioral testing was conducted by one experimenter (P.B.L.), who was blind to the lesion condition of the individual monkeys.

\section{Lesion evaluation}

After completion of behavioral testing, all experimentally lesioned monkeys and one sham-operated monkey were killed, and their brains were prepared for histological evaluation. Monkeys were deeply anesthetized with pentobarbital $(50 \mathrm{mg} / \mathrm{kg}$, i.v.) and perfused transcardially with 1 and $4 \%$ paraformaldehyde in $0.1 \mathrm{~m}$ phosphate buffer, $\mathrm{pH} 7.4$. The brains were postfixed for $6 \mathrm{~h}$ in the same fixative, cryoprotected in 10 and $20 \%$ glycerol solutions in $0.1 \mathrm{M}$ phosphate buffer, $\mathrm{pH} 7.4$ (for 24 and $72 \mathrm{~h}$, respectively), rapidly frozen in isopentane, and stored at $-70^{\circ} \mathrm{C}$ until sectioning. Sections were cut coronally at $30 \mu \mathrm{m}$ on a freezing, sliding microtome. A series of one-in-eight sections was mounted on gelatincoated slides and stained by the Nissl method with thionin.

Microscopic evaluation of the extent of the lesions was conducted on an AusJena microfiche reader (Zeiss, Oberkochen, Germany) and a Nikon Eclipse E600 (Nikon, Melville, NY). The boundaries of the subdivisions of the medial temporal lobe structures were microscopically determined following the nomenclature described previously (Lavenex and Amaral, 2000; Lavenex et al., 2002, 2004a,b; Amaral and Lavenex, 2006). All Nissl-stained sections ( $240 \mu \mathrm{m}$ apart) were first examined at low magnification (AusJena at $13 \times$ ). The extent of the lesion was evaluated for both the left and right side of the brain on every other section $(480 \mu \mathrm{m}$ apart) at higher magnification (Nikon Eclipse at $40 \times$ and $200 \times$ when necessary). Photomicrographs of representative coronal sections throughout the hippocampal formation were taken with a Spot digital camera system (Diagnostic Instruments, Sterling Heights, MI) connected to a Macintosh computer PowerMac G4 (Apple Computers, Cupertino, CA). Artifacts located outside the sections were removed, and levels were adjusted in Photoshop 8.0 (Adobe Systems, San Jose, CA).

\section{Results}

We first evaluated whether monkeys' choices were influenced by their memory of the locations that were baited during the previous session ( $24 \mathrm{~h}$ earlier). If monkeys remembered which array of three distinct locations was baited during the previous session and used this knowledge to guide their choices (i.e., to choose the same locations as had been baited during the previous session), we would expect the number of correct choices to be higher when the baited array was the same as during the previous session compared with when the alternate array had been baited during 
the previous session (Lavenex et al., 1998). Because the monkeys' choices could obviously be influenced by learning within a session (i.e., from trial 1, to trial 2, to trial 3), we considered only the first trial of each session for this analysis. Group and individual analyses did not reveal evidence that any sham-operated or hippocampal-lesioned monkey relied on its long-term (24 h) memory of the baited locations to guide its choices among potentially-baited locations in either the local cue or spatial relational condition (data not shown).

We also evaluated whether monkeys' choices were influenced by their memory of the locations that were baited during the previous trial(s) within a daily session ( 1 min intertrial interval). Monkeys performed three trials per session, during which the baited locations remained the same. If monkeys remembered which array of three distinct locations was baited during the previous trial and used this knowledge to guide their choices, we would expect the number of correct choices to increase within a daily session (Lavenex et al., 1998). Our analyses did not reveal evidence that any sham-operated or hippocampal-lesioned monkey relied on its short-term ( $1 \mathrm{~min}$ ) memory of the baited locations to guide its choices among potentially-baited locations in either the local cue or spatial relational condition (data not shown).

Finally, because three locations were always baited simultaneously, monkeys could also predict which array was baited after sampling one of the six potentially-baited locations (Lavenex and Banta Lavenex, 2006). Detailed analyses of group and individual behaviors did not reveal evidence that any sham-operated or hippocampal-lesioned monkey modified its behavior based on the first cup sampled (data not shown).

After determining that neither the monkeys' short-term (1 min) nor long-term (24 h) memory of the baited locations influenced their choices among potentially-baited locations, we evaluated the ability of, and the behavioral strategies exhibited by, hippocampal-lesioned and sham-operated monkeys to discriminate potentially-baited locations from never-baited locations in the local cue and spatial relational conditions.

\section{Local cue condition}

The analysis of the first choice and the first four choices made during an individual trial revealed no group differences in the local cue condition (first choice: group effect, $F_{(1,9)}=0.271$, $p=0.6151$; cup type, $F_{(4,36)}=35.613, p<0.0001$; interaction, $F_{(4,36)}=1.547, p=0.2095$; first four choices: group effect, $F_{(1,9)}=2.929, p=0.1212$; cup type, $F_{(4,36)}=193.789, p<0.0001$; interaction, $\left.F_{(4,36)}=1.215, p=0.3216\right)$.

Hippocampal-lesioned monkeys discriminated the potentially-baited locations marked by local cues (Fig. 2 A). For the first choice (i.e., the first cup opened during individual trials), hippocampal-lesioned monkeys chose more potentially-baited locations on the inner and outer arrays than never-baited locations $\left(F_{(4,20)}=25.652, p<0.0001\right.$; Pot IN $>$ Pot OUT $>$ Equ $\mathrm{IN}=$ Equ OUT $=$ Other; all $p<0.0053)$. Considering the first four choices (i.e., the first four cups opened during individual trials), hippocampal-lesioned monkeys continued to chose more potentially-baited locations on the inner and outer arrays than never-baited locations $\left(F_{(2,20)}=79.379, p<0.0001\right.$; Pot IN $>$ Pot $\mathrm{OUT}>$ Equ IN $>$ Equ OUT $=$ Other; all $p<0.035)$. Note that hippocampal-lesioned monkeys exhibited a preference for locations on the inner array for both the first choice (Pot IN > Pot OUT) and first four choices (Pot IN $>$ Pot OUT and Equ IN $>$ Equ OUT).

Not surprisingly, control monkeys also discriminated the
Hippocampals: Local Cue Condition
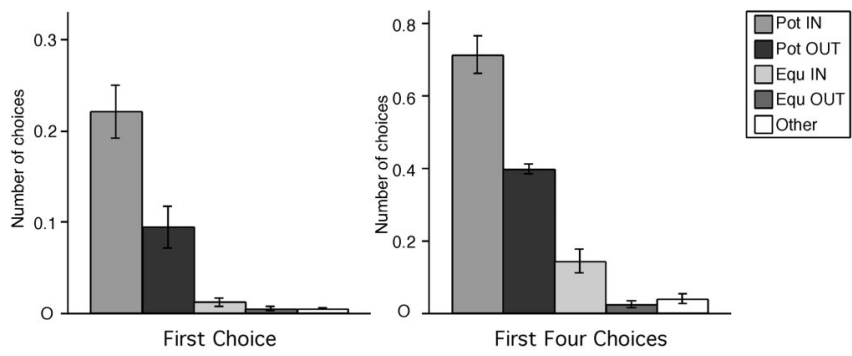

B

Controls: Local Cue Condition
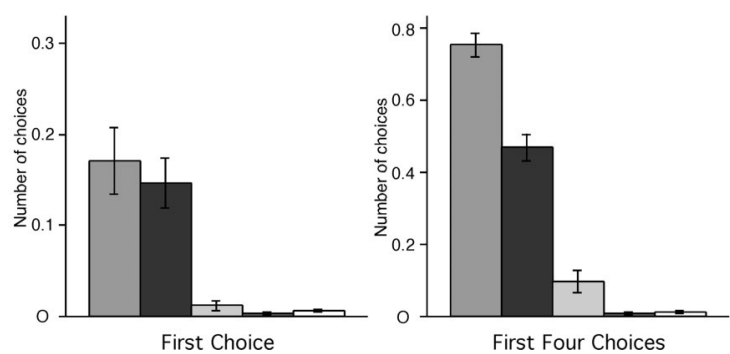

Figure 2. Hippocampal-lesioned $(\boldsymbol{A})$ and control $(\boldsymbol{B})$ monkeys' strategy in the local cue condition. Pot IN, Potentially-baited locations at the corners of the inner hexagon (locations 13, 15, 17); Pot OUT, potentially-baited locations at the corners of the outer hexagon (locations 4, 8 , 12); Equ IN, never-baited locations at the corners of the inner hexagon (locations 14, 16, 18); Equ OUT, never-baited locations at the corners of the outer hexagon (locations 2,6, 10); 0ther, never-baited locations on the sides of the outer hexagon (locations 1,3,5,7,9,11). The number of choices in each category $(n)$ is normalized according to the probability of making that choice ( $n$ of 3 for Pot IN, Pot OUT, Equ IN, and Equ OUT; $n$ of 6 for Other).

potentially-baited locations marked by local cues (Fig. 2 B). For their first choice, they chose more potentially-baited locations on the inner and outer arrays than never-baited locations; they did not exhibit a preference for locations on the inner array $\left(F_{(4,16)}=\right.$ 13.215, $p<0.0001$; Pot IN $=$ Pot OUT $>$ Equ IN $=$ Equ OUT $=$ Other; all $p<0.0009)$. For their first four choices, control monkeys still chose more potentially-baited locations on the inner and outer arrays than never-baited locations $\left(F_{(2,16)}=130.81\right.$, $p<0.0001$; all $p<0.0001$ ) but also demonstrated a preference for locations on the inner array (Pot IN $>$ Pot OUT, $p<0.0001$; Equ $\mathrm{IN}>$ Equ OUT, $p=0.0474$; Equ IN $=$ Other, $p=0.0541)$.

In summary, both hippocampal-lesioned and sham-operated control monkeys discriminated potentially-baited cups at fixed locations marked by local cues.

\section{Dissociation probe trials}

To assess the monkeys' reliance on local cues versus spatial relational information to find the food locations in the local cue condition, they were tested on two probe trials (after each testing phase in the local cue condition), during which the locations of the colored cups were shifted $60^{\circ}$ from their usual spatial locations and no food was present (Fig. $1 B$ ).

\section{Probe trial 1}

The first dissociation probe trial (on day 16, after $15 \mathrm{~d}$ or 45 trials in the first phase of the local cue condition) revealed significant differences in the behavioral strategies exhibited by control and hippocampal-lesioned monkeys (first six choices: group effect, $F_{(1,9)}=1.733, p=0.2206$; cup type, $F_{(4,36)}=52.036, p<0.0001$; interaction, $F_{(4,36)}=1.253, p=0.3061$; all choices: group effect, $F_{(1,9)}=1.033, p=0.3360$; cup type, $F_{(4,36)}=52.260, p<0.0001$; interaction, $\left.F_{(4,36)}=3.123, p=0.0265\right)$. 
Probe Trial 1

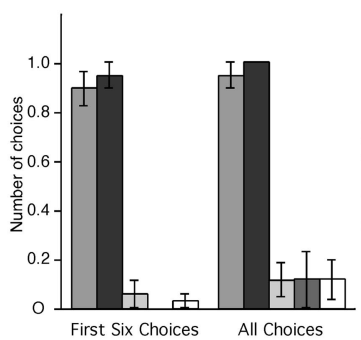

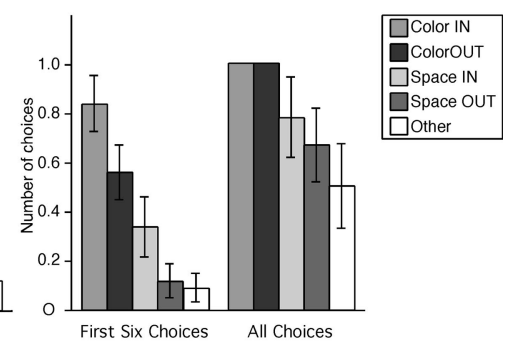

Controls
Probe Trial 1

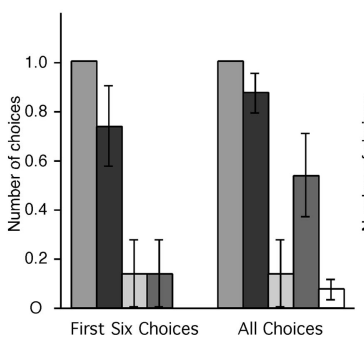

Probe Trial 2

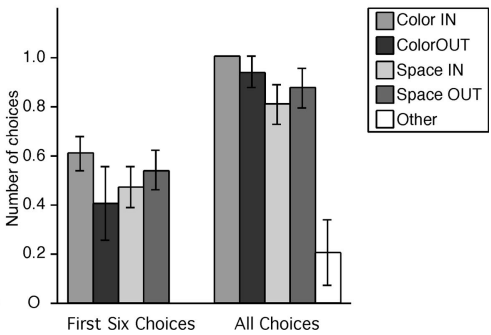

Figure 3. Hippocampal-lesioned $(\boldsymbol{A})$ and control $(\boldsymbol{B})$ monkeys' choices in the two dissociation probe trials (no food present). Color IN, Red cups at never-baited locations at the corners of the inner hexagon (locations 14, 16, 18); Color OUT, blue cups at never-baited locations at the corners of the outer hexagon (locations 2, 6, 10); Space IN, neutral cups at correct spatial locations at the corners of the inner hexagon (locations 13, 15, 17); Space 0UT, neutral cups at correct spatial locations at the corners of the outer hexagon (locations 4, 8, 10); 0ther, neutral cups at never-baited locations on the sides of the outer hexagon (locations 1,3,5,7,9,11). The number of choices in each category $(n)$ is normalized according to the probability of making that choice ( $n$ of 3 for Color IN, Color OUT, Space IN, and Space OUT; $n$ of 6 for Other).

Hippocampal-lesioned monkeys chose almost exclusively locations marked by local cues (Fig. $3 A$ ) (first six choices: $F_{(4,20)}=$ $86.14, p<0.0001$, Color IN $=$ Color OUT $>$ Space IN $=$ Space OUT $=$ Other; all $p<0.001$; all choices: $F_{(4,20)}=65.623, p<$ 0.0001 , Color IN $=$ Color OUT $>$ Space $\mathrm{IN}=$ Space OUT $=$ Other; all $p<0.001)$. Indeed, hippocampal-lesioned monkeys chose nearly every location marked by a local cue (they opened 35 of 36 colored cups across all monkeys). In contrast, they chose very few locations that were not marked by a local cue even after opening the six colored cups and not finding any food (they opened a total of only 8 of 72 neutral cups across all monkeys). In summary, hippocampal-lesioned monkeys relied exclusively on local cues to find the food locations and did not exhibit any evidence of spatial relational learning in the first phase of testing in the local cue condition.

For their first six choices, control monkeys also chose mostly locations marked by local cues (Fig. $3 B)\left(F_{(4,16)}=12.28, p<\right.$ 0.0001; Color IN $=$ Color OUT $>$ Space $\mathrm{IN}=$ Space OUT $=$ Other; all $p<0.004)$. For all choices, however, control monkeys chose mostly locations marked by local cues, but they also discriminated correct spatial locations on the outer array $\left(F_{(4,16)}=\right.$ 13.992, $p<0.0001$; Color IN $=$ Color OUT; Color IN $>$ Space IN; Color OUT $=$ Space OUT $>$ Other; all $p<0.01)$. Thus, although control monkeys relied preferentially on local cues to find the food locations in the first phase of training in the local cue condition (first six choices), they also exhibited evidence of spatial relational learning (all choices).
Probe trial 2

The second dissociation probe trial (on day 41, after $25 \mathrm{~d}$ and $15 \mathrm{~d}$ of experience in the local cue and spatial relational conditions, respectively) revealed additional information about the different behavioral strategies exhibited by control and hippocampallesioned monkeys (first six choices: group effect, $F_{(1,9)}=1.753$, $p=0.2181$; cup type, $F_{(4,36)}=12.448, p<0.0001$; interaction, $F_{(4,36)}=3.631, p=0.0138$; all choices: group effect, $F_{(1,9)}=$ $0.090, p=0.7709$; cup type, $F_{(4,36)}=13.022, p<0.0001$; interaction, $\left.F_{(4,36)}=1.604, p=0.1945\right)$.

For the first six choices, hippocampal-lesioned monkeys chose preferentially locations marked by local cues and failed to discriminate correct spatial locations $\left(F_{(4,20)}=8.331, p=0.0004\right.$; Color IN = Color OUT; Color IN $>$ Space IN; Color OUT $>$ Space OUT $=$ Other; all $p<0.01$ ). For all choices, hippocampallesioned monkeys chose more locations marked by local cues on the inner and outer arrays than correct spatial locations on the outer array $\left(F_{(4,20)}=3.821, p=0.0183\right.$; Color IN $>$ Space OUT; $p=0.0453$; Color OUT $>$ Space OUT; $p=0.0453$ ) and more locations marked by local cues on the inner and outer arrays than other, never-baited locations on the outer array (Color IN = Color OUT $>$ Other; all $p=0.0046$ ), confirming their preference for locations marked by local cues. In contrast to the first probe trial, hippocampal-lesioned monkeys opened a significant number of neutral cups during the second dissociation probe trial (they opened 44 of 72 neutral cups across all monkeys). However, they still did not discriminate between correct spatial locations and other, never-baited locations (Space IN $=$ Space OUT $=$ Other; all $p>0.0911)$. In summary, hippocampal-lesioned monkeys relied on local cues to find the food locations and did not exhibit any evidence of spatial relational learning after the second phase of testing in the local cue condition.

During the second probe trial, control monkeys, in contrast, chose locations marked by local cues and correct spatial locations with equal frequency, and more often than other, never-baited locations, for both the first six choices $\left(F_{(4,16)}=9.651\right.$, $p=0.0004$; Color IN $=$ Color OUT $=$ Space IN $=$ Space OUT $>$ Other; all $p<0.01)$ and for all choices made $\left(F_{(4,16)}=15.161\right.$, $p<0.0001$; Color IN $=$ Color OUT $=$ Space $\mathrm{IN}=$ Space OUT $>$ Other; all $p<0.0001)$. In summary, control monkeys relied equally on local cues and spatial information to find the food locations in the second phase of testing in the local cue condition, exhibiting additional evidence of spatial relational learning.

\section{Spatial relational condition}

The analysis of the first choice and the first four choices made during an individual trial revealed significant group differences in the spatial relational condition (first choice: group effect, $F_{(1,9)}=1.587, p=0.2395$; cup type, $F_{(4,36)}=19.229, p<0.0001$; interaction, $F_{(4,36)}=7.574, p=0.0002$; first four choices: group effect, $F_{(1,9)}=25.255, p=0.0007$; cup type, $F_{(4,36)}=105.677, p<$ 0.0001 ; interaction, $\left.F_{(4,36)}=13.507, p<0.0001\right)$.

Hippocampal-lesioned monkeys did not discriminate the potentially-baited cups in the absence of local cues marking their locations (Fig. 4A). For their first choice, hippocampal-lesioned monkeys exhibited a preference for locations on the inner array but did not discriminate between potentially-baited and neverbaited locations on the inner array $\left(F_{(4,20)}=14.771, p<0.0001\right.$; Pot IN $=$ Equ IN $>$ all others; all $p<0.0004)$, and neither did hippocampal-lesioned monkeys discriminate between potentially-baited and never-baited locations on the outer array $($ Pot OUT $=$ Equ OUT $=$ Other; all $p>0.23)$. With respect to their first four choices, hippocampal-lesioned monkeys still ex- 
A Hippocampals: Spatial Relational Condition
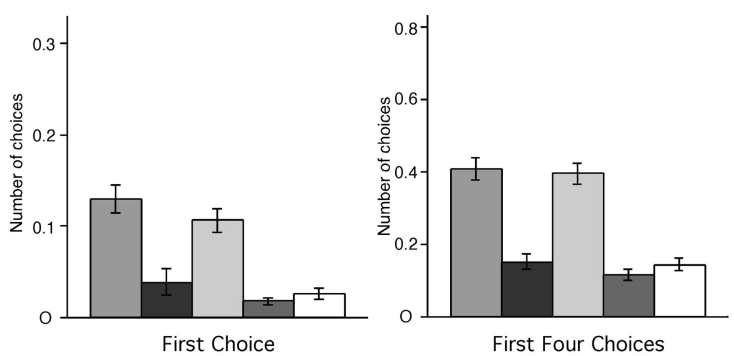

B
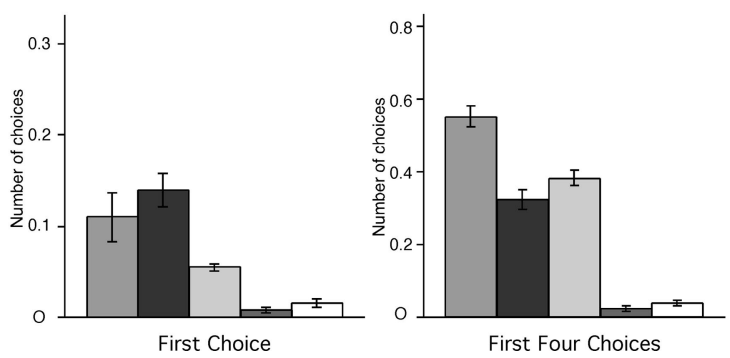

Figure 4. Hippocampal-lesioned $(\boldsymbol{A})$ and control $(\boldsymbol{B})$ monkeys' strategy in the spatial relational condition. For abbreviations, see Figure 2.

hibited a preference for locations on the inner array but continued to fail to discriminate between potentially-baited and neverbaited locations on the inner array $\left(F_{(4,20)}=32.187, p<0.0001\right.$; Pot IN $=$ Equ IN $>$ all others; all $p<0.0001)$. Again, hippocampal-lesioned monkeys did not discriminate between potentiallybaited and never-baited locations on the outer array (Pot OUT = Equ OUT $=$ Other; all $p>0.31$ ). In summary, although hippocampal-lesioned monkeys exhibited a preference for locations on the inner array, they did not exhibit any evidence of spatial relational learning in the spatial relational condition.

As predicted, control monkeys discriminated the potentiallybaited locations in absence of the local cues (Fig. 4B). For their first choice, control monkeys chose potentially-baited locations on both the inner and outer array more often than never-baited locations $\left(F_{(4,16)}=11.931, p=0.001\right.$; Pot IN $=$ Pot OUT $>$ Equ $\mathrm{IN}=$ Equ OUT $=$ Other; all $p<0.04)$. For their first four choices, control monkeys chose potentially-baited locations on the inner array more often than any other location. $\left(F_{(4,16)}=99.691, p<\right.$ 0.0001; Pot IN $>$ all others; all $p<0.0001)$ and chose potentiallybaited locations on the outer array and never-baited locations on the inner array with equal frequency, but more often than neverbaited locations on the outer array (Pot OUT $=$ Equ IN $>$ Equ OUT $=$ Other; all $p<0.0001)$. It is important to recognize that, although control monkeys demonstrated a preference for locations on the inner array, they still discriminated between potentially-baited and never-baited, topologically equivalent locations on the inner array (Pot IN $>$ Equ IN; $p<0.0001$ ) and between potentially-baited and never-baited, topologically equivalent locations on the outer array (Pot OUT > Equ OUT; $p<0.001$ ). In summary, control monkeys exhibited evidence of spatial relational learning in the spatial relational condition.

\section{Specific lack of spatial relational memory}

Finally, to determine whether monkeys' behavioral strategies were affected by continual testing, we analyzed their first four choices during the four alternating phases of testing separately: weeks $2-3$ in the local cue condition; weeks $5-6$ in the spatial relation condition; weeks $7-8$ in the local cue condition; and weeks 9-10 in the spatial relational condition. The analysis of the first four choices in the first phase of testing in the local cue conditions (weeks 2-3) did not reveal any group difference, indicating that task acquisition was similar between lesion conditions (group effect, $F_{(1,9)}=0.137, p=0.7201$; cup type, $F_{(4,36)}=$ 243.945, $p=0.0001$; interaction, $\left.F_{(4,36)}=0.092, p=0.9845\right)$.

Hippocampal-lesioned monkeys discriminated the potentially-baited locations only when local cues marked these locations (weeks 2-3: $F_{(4,20)}=125.763, p<0.0001$, Pot IN $>$ Pot OUT $>$ Equ IN $=$ Equ OUT $=$ Other; weeks 7-8: $F_{(4,20)}=$ $50.277, p<0.0001$, Pot IN $>$ Pot OUT $>$ Equ IN $>$ Equ OUT $=$ Other) but not in the absence of local cues (weeks 5-6: $F_{(4,20)}=16.188, p<0.0001$, Pot IN $=$ Equ IN $>$ Pot OUT $=$ Equ OUT $=$ Other; weeks 9-10: $F_{(4,20)}=37.687, p<0.0001$, Pot $\mathrm{IN}=$ Equ IN $>$ Pot OUT $=$ Equ OUT $=$ Other $).$ In contrast, control monkeys discriminated potentially-baited locations in the presence of local cues marking these locations (weeks 2-3: $F_{(4,16)}=123.678, p<0.0001$, Pot IN $>$ Pot OUT $>$ Equ IN $=$ Equ OUT $=$ Other; weeks 7-8: $F_{(4,16)}=109.679, p<0.0001$, Pot IN $>$ Pot OUT $>$ Equ IN $>$ Equ OUT $=$ Other $)$, as well as in their absence (weeks 5-6: $F_{(4,16)}=86.831, p<0.0001$, Pot IN $>$ Equ $\mathrm{IN}>$ Pot $\mathrm{OUT}>$ Equ OUT $=$ Other; weeks $9-10, F_{(4,16)}=$ $61.954, p<0.0001$, Pot IN $>$ Pot OUT $=$ Equ IN $>$ Equ OUT $=$ Other). Altogether, these data establish the specific lack of spatial relational learning and memory in hippocampal-lesioned monkeys.

\section{Individual behaviors}

The behavioral strategy exhibited by each individual monkey was also analyzed to determine whether any adult monkey that received bilateral hippocampal lesion was capable of spatial relational learning. In summary, every sham-operated and hippocampal-lesioned monkey discriminated the potentiallybaited locations marked by local cues (Table 1). In contrast, none of the hippocampal-lesioned monkeys discriminated the potentially-baited locations in the absence of local cues (Table 2). One hippocampal-lesioned monkey (MMU26149) did, however, appear to discriminate potentially-baited locations and neverbaited locations on the outer array (Table 2), but this discrimination can be attributed to the animal's bias toward cup 12 located along the back wall of the apparatus. Detailed analysis of this monkey's choices on the inner array, as well as comparison between other potentially-baited locations (cups 4 and 8 ) and never-baited locations on the outer array, did not reveal any evidence of spatial relational learning.

Interestingly, only five of the six control monkeys discriminated the potentially-baited locations in the absence of local cues. The behavior of the sixth monkey, MMU26736, was indistinguishable from that of the experimentally lesioned monkeys. In the spatial relational condition, MMU26736 showed a preference for locations on the inner array but did not discriminate between potentially-baited and never-baited, topologically equivalent locations (Table 2). Histological analysis of this monkey's brain revealed bilateral, selective damage to the hippocampus (for a detailed description of the lesion, see below).

In summary, individual behavioral analyses demonstrate the consistent inability of hippocampal-lesioned monkeys to establish or use an allocentric (spatial relational) representation of the environment. 
Table 1. First four choices in the local cue condition, individual behaviors

\begin{tabular}{|c|c|c|c|c|c|}
\hline & Pot IN & Pot OUT & Equ IN & Equ OUT & Other \\
\hline \multicolumn{6}{|c|}{ Hippocampal-lesioned } \\
\hline MMU26544 & $0.846 \pm 0.024$ & $0.382 \pm 0.038$ & $0.061 \pm 0.022$ & $0.011 \pm 0.008$ & $0.009 \pm 0.005$ \\
\hline MMU26669 & $0.730 \pm 0.028$ & $0.382 \pm 0.037$ & $0.138 \pm 0.035$ & $0.011 \pm 0.008$ & $0.039 \pm 0.010$ \\
\hline MMU28086 & $0.702 \pm 0.037$ & $0.422 \pm 0.052$ & $0.149 \pm 0.035$ & $0.000 \pm 0.000$ & $0.029 \pm 0.009$ \\
\hline MMU27645 & $0.550 \pm 0.029$ & $0.336 \pm 0.019$ & $0.242 \pm 0.023$ & $0.028 \pm 0.014$ & $0.090 \pm 0.012$ \\
\hline MMU26149 & $0.857 \pm 0.024$ & $0.427 \pm 0.032$ & $0.039 \pm 0.020$ & $0.006 \pm 0.006$ & $0.000 \pm 0.000$ \\
\hline MMU26296 & $0.569 \pm 0.036$ & $0.397 \pm 0.040$ & $0.194 \pm 0.028$ & $0.065 \pm 0.019$ & $0.047 \pm 0.014$ \\
\hline Average & $0.709 \pm 0.054$ & $0.391 \pm 0.014$ & $0.137 \pm 0.032$ & $0.020 \pm 0.010$ & $0.036 \pm 0.013$ \\
\hline \multicolumn{6}{|l|}{ Controls } \\
\hline MMU26922 & $0.702 \pm 0.026$ & $0.585 \pm 0.033$ & $0.028 \pm 0.014$ & $0.011 \pm 0.008$ & $0.003 \pm 0.003$ \\
\hline MMU26734 & $0.797 \pm 0.026$ & $0.471 \pm 0.031$ & $0.066 \pm 0.022$ & $0.000 \pm 0.000$ & $0.000 \pm 0.000$ \\
\hline MMU28056 & $0.647 \pm 0.025$ & $0.399 \pm 0.028$ & $0.193 \pm 0.028$ & $0.017 \pm 0.009$ & $0.029 \pm 0.011$ \\
\hline MMU26701 & $0.802 \pm 0.026$ & $0.489 \pm 0.033$ & $0.044 \pm 0.017$ & $0.000 \pm 0.000$ & $0.000 \pm 0.000$ \\
\hline MMU25920 & $0.802 \pm 0.026$ & $0.376 \pm 0.025$ & $0.132 \pm 0.029$ & $0.000 \pm 0.000$ & $0.012 \pm 0.006$ \\
\hline Average & $0.750 \pm 0.032$ & $0.464 \pm 0.037$ & $0.093 \pm 0.031$ & $0.006 \pm 0.004$ & $0.009 \pm 0.006$ \\
\hline MMU26736 & $0.674 \pm 0.035$ & $0.399 \pm 0.028$ & $0.121 \pm 0.026$ & $0.050 \pm 0.017$ & $0.046 \pm 0.013$ \\
\hline \multicolumn{6}{|c|}{ 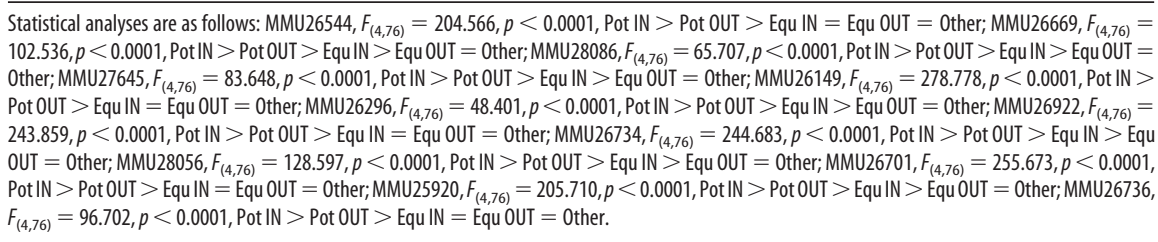 } \\
\hline
\end{tabular}

Table 2. First four choices in the spatial relational condition, individual behaviors

\begin{tabular}{|c|c|c|c|c|c|}
\hline & Pot IN & Pot OUT & Equ IN & Equ OUT & Other \\
\hline \multicolumn{6}{|c|}{ Hippocampal-lesioned } \\
\hline MMU26544 & $0.495 \pm 0.024$ & $0.066 \pm 0.022$ & $0.445 \pm 0.030$ & $0.088 \pm 0.013$ & $0.122 \pm 0.013$ \\
\hline MMU26669 & $0.364 \pm 0.027$ & $0.149 \pm 0.022$ & $0.393 \pm 0.025$ & $0.077 \pm 0.018$ & $0.175 \pm 0.011$ \\
\hline MMU28086 & $0.445 \pm 0.037$ & $0.111 \pm 0.016$ & $0.405 \pm 0.030$ & $0.121 \pm 0.019$ & $0.124 \pm 0.015$ \\
\hline MMU27645 & $0.341 \pm 0.014$ & $0.193 \pm 0.016$ & $0.297 \pm 0.018$ & $0.143 \pm 0.018$ & $0.177 \pm 0.011$ \\
\hline MMU26149 & $0.467 \pm 0.032$ & $0.171 \pm 0.027$ & $0.484 \pm 0.040$ & $0.072 \pm 0.023$ & $0.070 \pm 0.017$ \\
\hline MMU26296 & $0.310 \pm 0.036$ & $0.187 \pm 0.020$ & $0.314 \pm 0.026$ & $0.160 \pm 0.031$ & $0.178 \pm 0.012$ \\
\hline Average & $0.404 \pm 0.031$ & $0.146 \pm 0.020$ & $0.390 \pm 0.030$ & $0.110 \pm 0.015$ & $0.141 \pm 0.018$ \\
\hline \multicolumn{6}{|l|}{ Controls } \\
\hline MMU26922 & $0.478 \pm 0.032$ & $0.411 \pm 0.033$ & $0.314 \pm 0.029$ & $0.039 \pm 0.014$ & $0.046 \pm 0.012$ \\
\hline MMU26734 & $0.518 \pm 0.030$ & $0.254 \pm 0.035$ & $0.431 \pm 0.029$ & $0.028 \pm 0.011$ & $0.052 \pm 0.011$ \\
\hline MMU28056 & $0.540 \pm 0.026$ & $0.281 \pm 0.017$ & $0.403 \pm 0.021$ & $0.011 \pm 0.008$ & $0.048 \pm 0.014$ \\
\hline MMU26701 & $0.648 \pm 0.021$ & $0.343 \pm 0.031$ & $0.336 \pm 0.026$ & $0.000 \pm 0.000$ & $0.003 \pm 0.003$ \\
\hline MMU25920 & $0.564 \pm 0.023$ & $0.292 \pm 0.017$ & $0.397 \pm 0.019$ & $0.017 \pm 0.009$ & $0.033 \pm 0.007$ \\
\hline Average & $0.550 \pm 0.028$ & $0.316 \pm 0.028$ & $0.376 \pm 0.022$ & $0.019 \pm 0.007$ & $0.036 \pm 0.009$ \\
\hline MMU26736 & $0.314 \pm 0.024$ & $0.187 \pm 0.024$ & $0.336 \pm 0.022$ & $0.184 \pm 0.021$ & $0.155 \pm 0.013$ \\
\hline
\end{tabular}

Statistical analyses are as follows: MMU26544, $F_{(4.76)}=77.947, p<0.0001$, Pot IN $=$ Equ IN $>$ Pot OUT $=$ Equ OUT $=$ Other; MMU26669, $F_{(4,76)}=34.251$ $p<0.0001$, Pot IN $=$ Equ IN $>$ Pot OUT $=0$ ther $>$ Equ 0UT; MMU28086, $F_{(476)}=37.402, p<0.0001$, Pot IN $=$ Equ IN $>$ Pot OUT $=$ Equ 0UT $=0$ 0ther; MMU27645, $F_{(4,76)}=24.329, p<0.0001$, Pot IN $=$ Equ IN $>$ Pot OUT = Equ OUT $=0$ ther; MMU26149, $F_{(4,76)}=41.856, p<0.0001$, Pot IN $=$ Equ IN $>$ Pot OUT $>$ Equ OUT $=$ Other; MMU26296, $F_{(4,76)}=6.636, p=0.0001$, Pot IN $=$ Equ IN $>$ Pot OUT $=$ Equ OUT $=$ Other; MMU26922, $F_{(4,76)}=51.015, p<$ 0.0001, Pot IN $=$ Pot OUT $>$ Equ IN $>$ Equ OUT $=0$ 0ther; MMU26734, $F_{(4,76)}=59.674, p<0.0001$, Pot IN $>$ Equ IN $>$ Pot OUT $>$ Equ 0UT $=0$ 0ther; MMU28056, $F_{(4,76)}=127.741, p<0.0001$, Pot IN $>$ Equ IN $>$ Pot OUT $>$ Equ 0UT $=0$ ther; MMU26701, $F_{(4,76)}=140.695, p<0.0001$, Pot IN $>$ Equ IN $=$ Pot OUT $>$ Equ OUT $=$ Other; MMU25920, $F_{(4,76)}=176.626, p<0.0001$, Pot IN $>$ Equ IN $>$ Pot OUT $>$ Equ OUT $=0$ 0ther; MMU26736, $F_{(4,76)}=12.339$, $p<0.0001$, Pot IN $=$ Equ IN $>$ Pot OUT $=$ Equ 0UT $=0$ ther.

\section{Lesion evaluation}

After completion of the behavioral experiment, all hippocampallesioned monkeys were killed, and their brains were processed for histological evaluation of the lesion. Experimental lesions were intended to selectively damage the dentate gyrus, hippocampus, subiculum, presubiculum, and parasubiculum and spare the adjacent entorhinal, perirhinal, and parahippocampal cortices. We describe the extent of the lesion for each experimentally lesioned monkey. Representative coronal sections at four different rostrocaudal levels are provided to illustrate the hippocampal formation of each lesioned case (Fig. $5 A-X$ ), as well as one nonlesioned case (Fig. 5Y-AB). The lesions were generally highly symmetrical, so that the illustrations are representative of both sides of the

\section{MMU28086}

brain (significant differences are described in the text). We did not attempt to provide quantitative evaluation of the damaged areas, because such information is not pertinent to the interpretation of the results and the conclusions of the study.

\section{MMU26544}

The entorhinal and perirhinal cortices were primarily intact on both sides of the brain, with the exception of very minor damage in the deep layers of the caudal limiting field (ECL) and area 35 (at the level of the uncal region) (Fig. 5A-D). The dentate gyrus, CA3, CA2, CA1, and subiculum were extensively damaged, showing some preservation only in the uncal region and the most rostral portion of the fields (for $\sim 2 \mathrm{~mm}$ from the genu hippocampus). There was near complete damage of these fields at more caudal levels, with the exception of minor preservation of the lateral part of the dentate gyrus and distal CA3 at midrostrocaudal levels. There was also very extensive damage of the presubiculum, parasubiculum, and areas TH, TFm, and TF1 of the parahippocampal cortex. The portion of the visual cortex area V4 located directly below the hippocampus was also extensively damaged.

\section{MMU26669}

The entorhinal and perirhinal cortices were primarily intact on both sides of the brain, with the exception of very minor damage in the deep layers of area 36c (Fig. $5 E-H)$. Lesion of the dentate gyrus, CA3, CA2, CA1, and the subiculum was nearly complete, with the exception of their uncal portion and the most rostral portion of the subiculum (at the genu of the hippocampus). Lesion of the presubiculum was nearly complete, whereas a significant portion of the caudal parasubiculum was preserved. In the parahippocampal cortex, area TFm was extensively damaged, whereas areas $\mathrm{TH}$ and $\mathrm{TFl}$ were more largely preserved. The portion of area V4 located directly below the hippocampus was extensively damaged.

The entorhinal cortex was intact on both sides of the brain (Fig. $5 I-L)$. Area 36 of the perirhinal cortex was also primarily intact, whereas area 35 was severely damaged in both hemispheres. Lesion of areas CA3, CA2, and CA1, as well as the subiculum was nearly complete. The dentate gyrus was severely damaged with the exception of its medial portion at midrostrocaudal levels. The uncal region was primarily intact. Areas TFm and TFl of the parahippocampal cortex were severely damaged, whereas area $\mathrm{TH}$ and the parasubiculum were mainly preserved. On the right side, a significant portion of area TEO [from the border with TFl and the fundus of the occipito-temporal sulcus (OTS)] was le- 
sioned. The portion of area V4 located directly below the hippocampus was heavily damaged.

\section{MMU27645}

The entorhinal and perirhinal cortices were primarily intact on both sides of the brain, with the exception of very minor damage in the deep layers of areas ECL, 35 and $36 \mathrm{~cm}$ (Fig. $5 M-P)$. The dentate gyrus, CA3, CA2, CA1, subiculum, presubiculum, and parasubiculum were nearly completely lesioned, with the exception of the most rostral portion of these fields and the uncal region. Areas TFm and TFl of the parahippocampal cortex were severely damaged, with the exception of the more superficial layers in the caudal portion of these fields. The rostral half of area TH was lesioned, whereas the caudal half was primarily preserved. The medial portion of area TEO (from the border with TFl to the fundus of OTS) and the portion of area V4 located directly below the hippocampus were severely damaged.

\section{MMU26149}

The entorhinal and perirhinal cortices were intact on both sides of the brain (Fig. $5 Q-T)$. The dentate gyrus, CA3, CA2, CA1, and the subiculum were completely lesioned (except for the uncal portion of these fields and the caudomedial portion of the dentate gyrus). The presubiculum and parasubiculum were partially lesioned, with a greater preservation of the parasubiculum on the right side. Area TFm of the parahippocampal cortex was nearly completely lesioned, whereas areas TH and TFl were only partially damaged. The portion of area V4 located directly below the hippocampus was heavily damaged.

\section{MMU26296}

The entorhinal and perirhinal cortices were primarily intact on both sides of the brain, with the exception of very minor damage in the deep layers of areas ECL and 35 (Fig. 5U-X). The deep layers of area 36c

were also slightly damaged on the right side. The dentate gyrus, CA3, CA2, CA1, and subiculum were extensively damaged, showing some preservation only in the uncal region and the most rostral part of their fields (for $\sim 2 \mathrm{~mm}$ from the genu hippocampus). There was near complete lesion of these fields at more caudal levels, with the exception of some minor preservation of the medial portion of the dentate gyrus at mid-rostrocaudal levels. There was significant damage of the presubiculum, parasubiculum, and area TH. Areas TFm and TFl of the parahippocampal cortex were damaged throughout their entire rostrocaudal extent. The medial portion of area TEO (from the border with TFl to the fundus of OTS) was damaged on the left side of the brain. The portion of area V4 located directly below the hippocampus was nearly completely lesioned. panels).

\section{B}
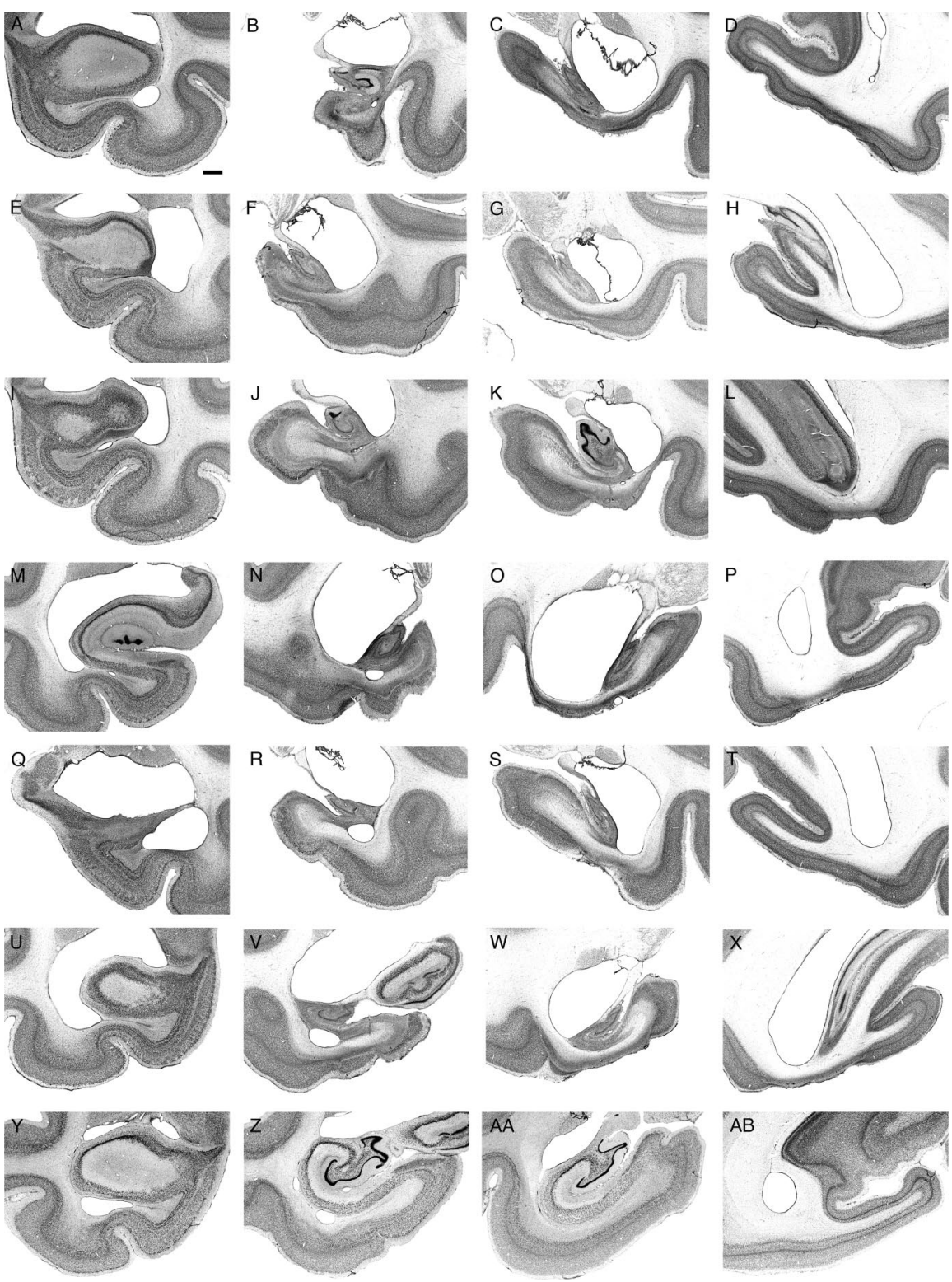

Figure 5. Photomicrographs illustrating the extent of the lesioned area in each experimentally lesioned monkey at four MMU28086; M-P, MMU27645; Q-T, MMU26149; U-X, MMU26296; $\boldsymbol{Y}-\mathbf{A B}$, nonlesioned monkey. Scale bar, $1 \mathrm{~mm}$ (applies to all

\section{Summary of experimental lesions}

Lesion extent was very consistent across all six monkeys. The entorhinal and perirhinal cortices were primarily, if not completely, intact in all experimentally lesioned monkeys. The intended targets, including the dentate gyrus, CA3, CA2, CA1, subiculum, presubiculum, and parasubiculum were always very significantly damaged, if not completely eliminated. However, the damaged areas also extended below the intended targets and included significant portions of the parahippocampal cortex, area TFm in particular. The medial portion of area TEO (from the border with area TFl to the fundus of the OTS) sustained restricted damage in some of the monkeys. The portion of area V4 located directly below the hippocampus was damaged in all monkeys. 

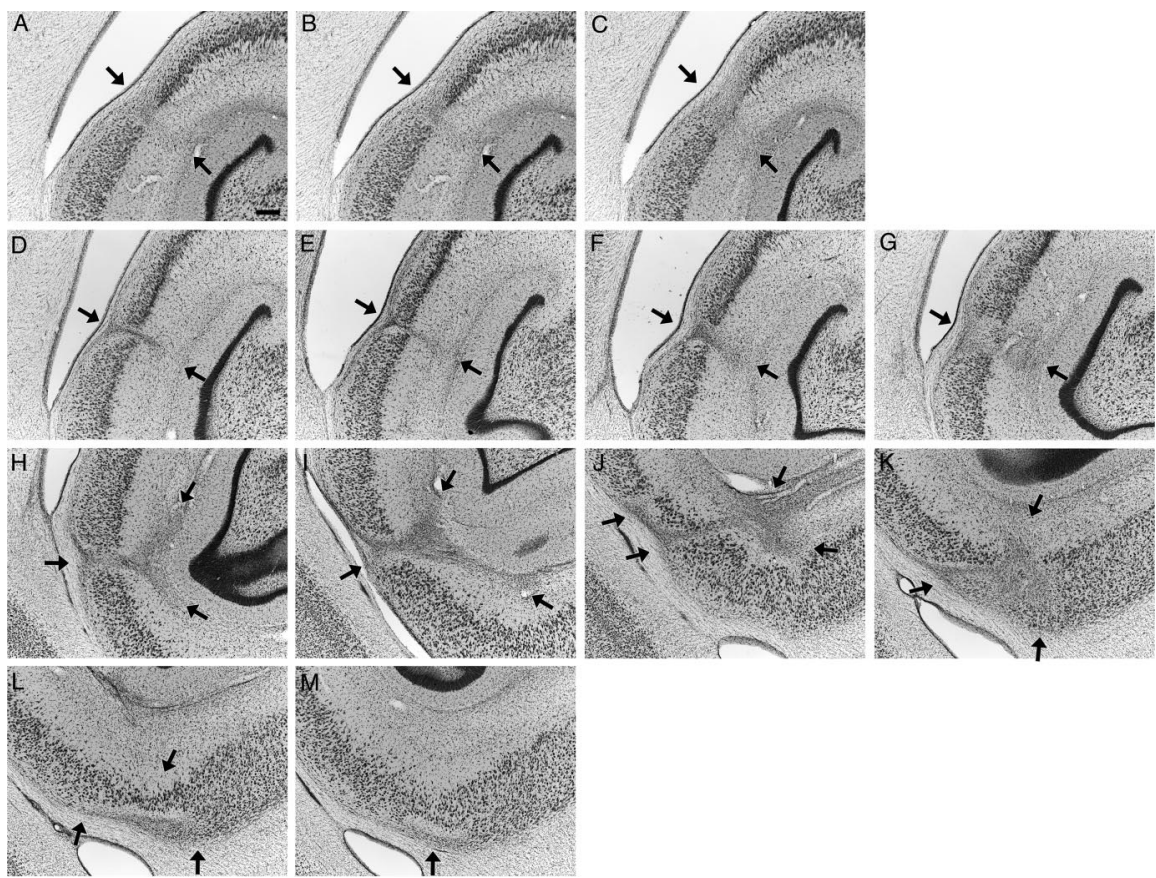

Figure 6. Photomicrographs illustrating the extent of the lesioned area (arrows) on the left side of the brain in the control monkey, MMU26736, whose behavior was identical to that of experimentally lesioned monkeys. $\boldsymbol{A}-\boldsymbol{C}$, Rostral area exhibiting neuronal damage, gliosis, and neuropil disorganization. $D-M$, Caudal area exhibiting neuronal damage, gliosis, and neuropil disorganization. Individual panels represent adjacent sections separated by $240 \mu \mathrm{m}$. All Nissl-stained sections on which signs of damage were visible are presented. There was an area of $\sim 1.44 \mathrm{~mm}$ between $\boldsymbol{C}$ and $\boldsymbol{D}$, with no visible neuron loss, gliosis, or disorganization of the neuropil. Scale bar, $250 \mu \mathrm{m}$ (applies to all panels).

\section{MMU26736}

As described above, this control monkey exhibited behavioral strategies identical to those of the experimentally lesioned monkeys. Histological examination of its brain revealed a bilateral lesion restricted to the hippocampus (Figs. 6, 7). Based on experience with experimentally induced ischemia (ZolaMorgan et al., 1992), we would suggest that this restricted lesion may have been induced by a transient ischemic episode at some point in the animal's life.

Similar to the experimentally lesioned monkeys, the entorhinal and perirhinal cortices were completely intact on both sides of the brain. In contrast to the experimentally lesioned monkeys, the dentate gyrus, subiculum, presubiculum, parasubiculum, parahippocampal cortex, and area V4 were also entirely preserved. Only the CA3, CA2, and CA1 fields of the hippocampus exhibited neuronal damage. Careful examination of the rest of the brain did not reveal any other damage or abnormalities. We describe in detail the abnormalities observed in both the left and right hippocampi.

In this monkey, the hippocampus (fields CA3, CA2, and CA1) extended $\sim 14.40 \mathrm{~mm}$ rostrocaudally. On the left side (Fig. 6), pyramidal neuron loss was visible at the CA2/CA1 border starting $\sim 2.64 \mathrm{~mm}$ caudally from the most rostral aspect of these fields. This narrow band exhibiting neuronal damage, gliosis, and cell reorganization extended caudally for $\sim 0.72$ $\mathrm{mm}$ (Fig. $6 A-C$ ). There was a region of $\sim 1.44 \mathrm{~mm}$ with no visible neuron loss, gliosis, or disorganization of the neuropil. Another area with pyramidal neuron loss and glial cell proliferation was visible at the CA2/CA1 border, $4.80 \mathrm{~mm}$ from the rostral part of the hippocampus. This second damaged area exhibiting cellular and neuropil reorganization extended caudally for $\sim 2.40 \mathrm{~mm}$, while moving progressively toward the

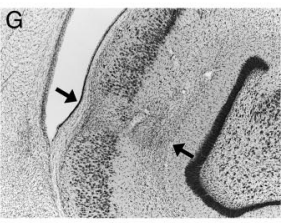

CA1/subiculum border (Fig. 6D-M) Together, these two areas of cell loss occupied $\sim 3.12 \mathrm{~mm}$ or $21 \%$ of the entire rostrocaudal extent of the left hippocampus.

The damage was more extensive on the right side (Fig. 7) and was also subdivided into two separate zones. The first area with extensive neuron loss and gliosis started $\sim 4.32 \mathrm{~mm}$ caudal from the rostral part of the hippocampus. At this level, there was a complete loss of the CA2 region (Fig. $7 A, B)$. Moving $240 \mu \mathrm{m}$ caudally, neuron loss extended both medially into the distal portion of CA3 (Fig. 7C,D) and laterally into proximal CA1 (Fig. $7 C, E$ ). This area exhibiting neuronal loss, gliosis, and cell reorganization extended caudally for another $1.20 \mathrm{~mm}$, moving progressively toward the CA1/subiculum border (Fig. $7 F-$ $J)$. There was then $\sim 4.08 \mathrm{~mm}$ with no visible neuron loss, gliosis, or disorganization of the neuropil. Neuron loss and gliosis was again visible at the CA2/CA1 border, at $\sim 10.84 \mathrm{~mm}$ from the rostral pole of the hippocampus (Fig. $7 K$ ). This second area exhibiting cellular and neuropil reorganization remained at the CA2/CA1 border for $\sim 1.92 \mathrm{~mm}$ (Fig. $7 K-R$ ). It extended caudally for another $1.20 \mathrm{~mm}$, moving progressively toward the CA1/ subiculum border (Fig. $7 S-W$ ). Together, these two areas of neuronal loss occupied $\sim 4.56 \mathrm{~mm}$ or $31 \%$ of the entire rostrocaudal extent of the right hippocampus.

These descriptions and illustrations indicate significant, bilateral pyramidal neuron loss, gliosis, and neuropil reorganization restricted to the hippocampus (fields CA3, CA2, and CA1).

\section{Discussion}

This study demonstrates that the adult monkey hippocampus is critical for the establishment or use of allocentric spatial representations. In this Discussion, we will consider three essential points: the cues used to guide monkeys' behavior, the underlying spatial representations, and the neuroanatomical structures subserving these behaviors and memory representations.

\section{Spatial relational learning}

In the local cue condition, hippocampal-lesioned and control monkeys readily discriminated the two sets of potentially-baited locations marked by different local cues. The first dissociation probe trial revealed that hippocampal-lesioned monkeys relied entirely on the local cues to guide their choices. Control monkeys, in contrast, although relying preferentially on the local cues to guide their choices (first six choices), demonstrated that they had also learned the potentially-baited locations in relation to distant environmental cues (all choices), even in the presence of local cues. The second probe trial revealed that, after $40 \mathrm{~d}$ or 120 trials of testing with potentially-baited cups in fixed locations, control monkeys relied equally on the local cues and the spatial relational information to guide their choices, whereas hippocampallesioned monkeys continued to rely entirely on the local cues to guide their choices.

We did not attempt to determine what distant cues were used 
by control monkeys to establish an allocentric representation of the environment (O'Keefe and Nadel, 1978; Schenk et al., 1995). We did, however, eliminate the possible use of uncontrolled local cues by rotating the board on which the cups were located between trials (Lavenex and Schenk, 1995, 1996, 1997, 1998; Lavenex et al., 1998; Lavenex and Banta Lavenex, 2006). More importantly, our experimental design precluded the reliance on an egocentric representation of space by alternating pseudorandomly between four different entrances into the open-field arena (O'Keefe and Nadel, 1978; Morris, 1981; Lavenex and Schenk, 1995, 1996, 1997, 1998; Schenk et al., 1995; Lavenex et al., 1998; Eichenbaum, 2000; Lavenex and Banta Lavenex, 2006) and using multiple goal locations (Lavenex and Banta Lavenex, 2006). To use an egocentric strategy in the spatial relational condition, monkeys would have to learn 24 separate egocentric coordinates (six locations from four different starting points). Furthermore, monkeys would have to return to one of the four entrance points after each choice to identify the location of the next goal to visit, a behavior that was never observed in our experiment. In contrast, monkeys were freely moving in the openfield arena, visiting one location after another without returning to an entrance point. In these conditions, control monkeys were able to discriminate potentiallybaited locations based on their relations with distant environmental cues, whereas hippocampal-lesioned monkeys were unable to do so.

Interestingly, both control and hippocampal-lesioned monkeys exhibited a preference for cups located on the inner array. This suggests that hippocampal-lesioned monkeys were able to organize their foraging from the center of the board to minimize travel distance (Lavenex and Banta Lavenex, 2006). This resulted in the opening of a proportionally higher number of cups located on the inner array, which were more easily reached from the center. However, in contrast to control monkeys, hippocampal-lesioned monkeys did not discriminate potentially-baited locations from topologically equivalent, never-baited locations on the same array, based on their relations with distant environmental cues. The primary and critical finding of this study, therefore, is that no hippocampal-lesioned monkey was able to rely on an allocentric spatial representation to navigate in the environment and guide its choices.

\section{Hippocampal lesion and spatial representations}

Experiments in rodents have shown that lesion of the hippocampus results in the consistent impairment of spatial relational learning and memory (Morris et al., 1982; Nadel, 1991; Schenk et al., 1995). In contrast, studies on the role of the monkey hippocampal formation in spatial learning and memory have resulted in inconsistent results. Although some studies have shown that
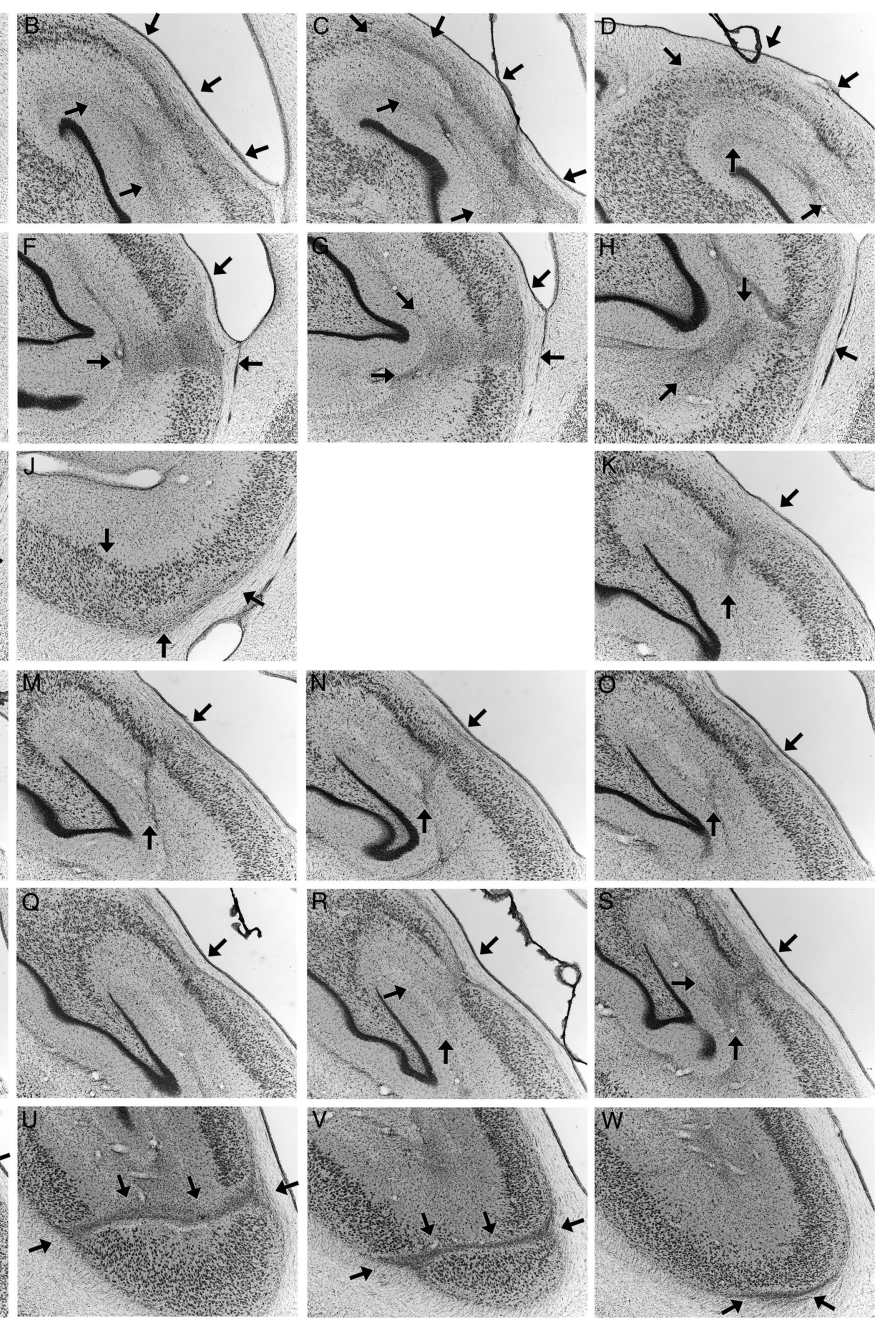

Figure 7. Photomicrographs illustrating the extent of the lesioned area (arrows) on the right side of the brain in the control lisorgaiza disorganization. Individual panels represent adjacent sections separated by $240 \mu \mathrm{m}$. All Nissl-stained sections on which signs of damage were visible are presented. There was an area of $\sim 4.08 \mathrm{~mm}$ between J and K, with no visible neuron loss, gliosis or disorganization of the neuropil. Scale bar, $250 \mu \mathrm{m}$ (applies to all panels).

hippocampal-lesioned monkeys exhibit impaired performance in various spatial memory tasks (Angeli et al., 1993; Beason-Held et al., 1999; Hampton and Murray, 2004; Alvarado and Bachevalier, 2005), others have failed to show any spatial memory impairment (Ridley et al., 1997; Murray and Mishkin, 1998; Malkova and Mishkin, 2003). However, if the monkey hippocampal formation is integrally involved with the processing of spatial relational information, as is the case in rodents, damage to the hippocampal formation should reliably and consistently impair the monkeys' ability to exhibit behavioral strategies dependent on allocentric representations of space.

We propose that previous monkey hippocampal lesion experiments failed to abolish spatial learning because the tasks in these studies could be solved, at least in part, by using egocentric (hippocampus-independent) representations of space (O'Keefe and Nadel, 1978; Nadel and Hardt, 2004). Normal monkeys perform optimally by using a combination of egocentric and allocentric strategies. In contrast, although hippocampal-lesioned monkeys are incapable of using allocentric strategies, they can still rely on egocentric strategies. Thus, depending on the parameters of the tasks, the performance of hippocampal-lesioned monkeys may appear either unimpaired or only partially im- 
paired compared with controls. Germane to this discussion is a series of open-field experiments (Hampton and Murray, 2004) that allowed tethered monkeys to walk about in a large-scale environment. Monkeys with bilateral hippocampal excitotoxic lesion were tested in match-to-location tasks. Unfortunately, however, the experiments failed to (1) demonstrate the reliance on an allocentric representation of space in normal monkeys (experiments 3 and 4), (2) distinguish controls from experimentally lesioned monkeys (experiments 1,3, and 4), and (3) rule out the use of an egocentric strategy (experiment 4). Thus, although their study attempted to address issues of egocentric versus allocentric spatial processing, it remained inconclusive with respect to the role of the monkey hippocampal formation in the establishment or use of allocentric representations of space.

Researchers have also interpreted the results of experiments that require animals to solve a spatial task after incremental delays as indicating that animals with hippocampal lesions exhibit a time-dependent decrease in performance [Murray and Mishkin, 1998; Alvardo and Bachevalier, 2005; Hampton and Murray, 2004 (experiment 2)]. Although performance did decrease as delay increased in all of these experiments, hippocampal-lesioned monkeys were still capable of solving the tasks at short delays, a finding that is not consistent with the idea of the hippocampus as a primary substrate for the processing of allocentric spatial representations. Alternatively, we suggest that, over short delays, an egocentric representation of goal locations can sustain performance in tasks such as those cited above. With increasing delays, control monkeys likely benefit from relying on an allocentric representation of space, whereas hippocampal-lesioned monkeys are unable to do so (this study; see also Burgess et al., 2002). This interpretation is consistent with studies on rodent spatial navigation, showing that an egocentric representation of space can sustain accurate navigation only over short distances or short periods of time (Etienne et al., 1996).

\section{Hippocampus versus parahippocampal cortex}

As noted previously, one of the control monkeys (MMU26736) did not exhibit any evidence of spatial relational learning but instead exhibited behavior identical to that of the hippocampallesioned monkeys. The inclusion of this animal was fortuitous because extensive histological analysis of its brain indicated that it had a circumscribed lesion of portions of the hippocampus bilaterally. The cause of the lesion is unknown, and the animal had an unremarkable medical history. The location of the lesion in the CA fields is reminiscent of experimentally induced ischemic lesions (Zola-Morgan et al., 1992). Although the extent of the damage appears more restricted than in experimentally induced ischemic lesions (we do not observe a decrease in the volume of the CA fields), we suspect that this may have been the cause of the brain damage observed in this monkey. The finding that this animal was unable to discriminate the baited locations in the spatial relational condition supports the idea that damage entirely restricted to the CA fields of the hippocampus is sufficient to produce clinically significant memory impairments (ZolaMorgan et al., 1986). In contrast to previous studies, however, the restricted hippocampus lesion observed in our study did not simply produce an impairment but prevented spatial relational processing altogether. We can therefore demonstrate a causal link between selective hippocampal damage and the inability to establish or use allocentric spatial representations.

Our results might seem to contradict the results of a previous study (Malkova and Mishkin, 2003) suggesting that the parahippocampal cortex, rather than the hippocampus, is essential for spatial learning in monkeys. However, we believe that, in their study, selective hippocampal lesion failed to produce any impairment (and parahippocampal cortex lesion resulted in impaired performance) because the monkeys could remember the location of the objects presented among the three possible locations by relying solely on an egocentric representation of space (i.e., left, center, or right). Together, the results of these studies would suggest that the hippocampus is essential for allocentric spatial representations, whereas the parahippocampal cortex might be more directly involved with the processing of egocentric representations of space.

\section{Conclusion}

Our findings demonstrate that the adult monkey hippocampal formation is critical for the establishment or use of allocentric representations of space and suggest that selective damage of the hippocampus prevents spatial relational learning in adult nonhuman primates. This study supports the view that the central role of the hippocampal formation in spatial relational learning and memory is conserved among vertebrates.

\section{References}

Alvarado MC, Bachevalier J (2005) Selective neurotoxic damage to the hippocampal formation impairs performance of the transverse patterning and location memory tasks in rhesus macaques. Hippocampus 15:118-131.

Amaral DG, Lavenex P (2006) Hippocampal neuroanatomy. In: The hippocampus book (Amaral DG, Andersen P, Bliss T, Morris RGM, O’Keefe J, eds). Oxford: Oxford UP, in press.

Angeli SJ, Murray EA, Mishkin M (1993) Hippocampectomized monkeys can remember one place but not two. Neuropsychologia 31:1021-1030.

Astur RS, Taylor LB, Mamelak AN, Philpott L, Sutherland RJ (2002) Humans with hippocampus damage display severe spatial memory impairments in a virtual Morris water task. Behav Brain Res 132:77-84.

Beason-Held LL, Rosene DL, Killiany RJ, Moss MB (1999) Hippocampal formation lesions produce memory impairment in the rhesus monkey. Hippocampus 9:562-574.

Bohbot VD, Iaria G, Petrides M (2004) Hippocampal function and spatial memory: evidence from functional neuroimaging in healthy participants and performance of patients with medial temporal lobe resections. Neuropsychology 18:418-425.

Brown MW, Aggleton JP (2001) Recognition memory: what are the roles of the perirhinal cortex and hippocampus? Nat Rev Neurosci 2:51-61.

Buckmaster CA, Eichenbaum H, Amaral DG, Suzuki WA, Rapp PR (2004) Entorhinal cortex lesions disrupt the relational organization of memory in monkeys. J Neurosci 24:9811-9825.

Burgess N, Maguire EA, O’Keefe J (2002) The human hippocampus and spatial and episodic memory. Neuron 35:625-641.

Cohen NJ, Eichenbaum H (1993) Memory, amnesia, and the hippocampal system. Cambridge, MA: MIT.

Eichenbaum H (1999) The hippocampus, memory, and place cells: is it spatial memory or a memory space? Neuron 23:209-226.

Eichenbaum H (2000) A cortical-hippocampal system for declarative memory. Nat Rev Neurosci 1:41-50.

Etienne AS, Maurer R, Seguinot V (1996) Path integration in mammals and its interaction with visual landmarks. J Exp Biol 199:201-209.

Georges-Francois P, Rolls ET, Robertson RG (1999) Spatial view cells in the primate hippocampus: allocentric view not head direction or eye position or place. Cereb Cortex 9:197-212.

Hampton RR, Murray EA (2004) Selective hippocampal damage in rhesus monkeys impairs spatial memory in an open-field test. Hippocampus 14:808-818.

Hori E, Tabuchi E, Matsumura N, Tamura R, Eifuku S, Endo S, Nishijo H, Ono T (2003) Representation of place by monkey hippocampal neurons in real and virtual translocation. Hippocampus 13:190-196.

Lavenex P, Amaral DG (2000) Hippocampal-neocortical interaction: A hierarchy of associativity. Hippocampus 10:420-430.

Lavenex P, Banta Lavenex P (2006) Spatial relational memory in ninemonth-old macaque monkeys. Learn Mem 13:84-96. 
Lavenex P, Schenk F (1995) Influence of local environmental olfactory cues on place learning in rats. Physiol Behav 58:1059-1066.

Lavenex P, Schenk F (1996) Integration of olfactory information in a spatial representation enabling accurate arm choice in the radial arm maze. Learn Mem 2:299-319.

Lavenex P, Schenk F (1997) Olfactory cues potentiate learning of distant visuospatial information. Neurobiol Learn Mem 68:140-153.

Lavenex P, Schenk F (1998) Olfactory traces and spatial learning in rats. Anim Behav 56:1129-1136.

Lavenex P, Shiflett MW, Lee RK, Jacobs LF (1998) Spatial versus nonspatial relational learning in free-ranging fox squirrels (Sciurus niger). J Comp Psychol 112:127-136.

Lavenex P, Suzuki WA, Amaral DG (2002) Perirhinal and parahippocampal cortices of the macaque monkey: projections to the neocortex. J Comp Neurol 447:394-420.

Lavenex P, Suzuki WA, Amaral DG (2004a) Perirhinal and parahippocampal cortices of the macaque monkey: intrinsic projections and interconnections. J Comp Neurol 472:371-394.

Lavenex P, Lavenex PB, Amaral DG (2004b) Nonphosphorylated highmolecular-weight neurofilament expression suggests early maturation of the monkey subiculum. Hippocampus 14:797-801.

Malkova L, Mishkin M (2003) One-trial memory for object-place associations after separate lesions of hippocampus and posterior parahippocampal region in the monkey. J Neurosci 23:1956-1965.

Mishkin M, Vargha-Khadem F, Gadian DG (1998) Amnesia and the organization of the hippocampal system. Hippocampus 8:212-216.

Morris RG, Mayes AR (2004) Long-term spatial memory: introduction and guide to the special section. Neuropsychology 18:403-404.

Morris RGM (1981) Spatial localization does not require the presence of local cues. Learn Motiv 12:239-260.

Morris RGM, Garrud P, Rawlins JNP, Okeefe J (1982) Place navigation impaired in rats with hippocampal-lesions. Nature 297:681-683.

Murray EA, Mishkin M (1998) Object recognition and location memory in monkeys with excitotoxic lesions of the amygdala and hippocampus. J Neurosci 18:6568-6582.

Nadel L (1991) The hippocampus and space revisited. Hippocampus $1: 221-229$.

Nadel L, Hardt O (2004) The spatial brain. Neuropsychology 18:473-476.

O'Keefe J, Dostrovsky J (1971) The hippocampus as a spatial map. Preliminary evidence from unit activity in the freely-moving rat. Brain Res $34: 171-175$
O'Keefe J, Nadel L (1978) The hippocampus as a cognitive map. Oxford: Clarendon.

Ono T, Tamura R, Nakamura K (1991) The hippocampus and space: are there "place neurons" in the monkey hippocampus? Hippocampus 1:253-257.

Parslow DM, Rose D, Brooks B, Fleminger S, Gray JA, Giampietro V, Brammer MJ, Williams S, Gasston D, Andrew C, Vythelingum GN, Ioannou G, Simmons A, Morris RG (2004) Allocentric spatial memory activation of the hippocampal formation measured with fMRI. Neuropsychology 18:450-461.

Ridley RM, Pearson C, Maclean CJ, Hoyle C, Baker HF, Kershaw TR, Hodges $\mathrm{H}$ (1997) Learning impairment induced by lesion of the cal field of the primate hippocampus-attempts to ameliorate the impairment by transplantation of fetal cal tissue. Exp Brain Res 115:83-94.

Rolls ET, O’Mara SM (1995) View-responsive neurons in the primate hippocampal complex. Hippocampus 5:409-424.

Rolls ET, Miyashita Y, Cahusac PM, Kesner RP, Niki H, Feigenbaum JD, Bach L (1989) Hippocampal neurons in the monkey with activity related to the place in which a stimulus is shown. J Neurosci 9:1835-1845.

Schenk F, Grobéty M-C, Lavenex P, Lipp H-P (1995) Dissociation between basic components of spatial memory in rats. In: Behavioural Brain Research in Naturalistic and Semi-Naturalistic Settings. NATO ASI series, Series D, Behavioural and Social Sciences (Alleva E, Fasolo A, Lipp H-P, Nadel L, Ricceri L, eds), pp 277-300. Amsterdam: Kluwer.

Scoville WB, Milner B (1957) Loss of recent memory after bilateral hippocampal lesions. J Neurol Neurosurg Psychiatry 20:11-21.

Shelton AL, Gabrieli JDE (2004) Neural correlates of individual differences in spatial learning strategies. Neuropsychology 18:442-449.

Squire LR, Zola SM (1998) Episodic memory, semantic memory, and amnesia. Hippocampus 8:205-211.

Tulving E, Markowitsch HJ (1998) Episodic and declarative memory: Role of the hippocampus. Hippocampus 8:198-204.

Zola-Morgan S, Squire LR, Amaral DG (1986) Human amnesia and the medial temporal region enduring memory impairment following a bilateral lesion limited to field CAl of the hippocampus. J Neurosci 6:2950-2967.

Zola-Morgan S, Squire LR, Rempel NL, Clower RP, Amaral DG (1992) Enduring memory impairment in monkeys after ischemic damage to the hippocampus. J Neurosci 12:2582-2596. 